\title{
Method validation for 243 pesticides and environmental contaminants in meats and poultry by tandem mass spectrometry coupled to low-pressure gas chromatography and ultrahigh-performance liquid chromatography
}

\author{
Lijun Han, ${ }^{\mathrm{a}, \mathrm{b}}$ Yelena Sapozhnikova, ${ }^{\mathrm{a}, *}$ and Steven J. Lehotay ${ }^{\mathrm{a}}$ \\ ${ }^{\text {a }}$ US Department of Agriculture, Agricultural Research Service; Eastern Regional Research \\ Center; 600 East Mermaid Lane; Wyndmoor, PA 19038; USA \\ ${ }^{\mathrm{b}}$ College of Science, China Agricultural University; No. 2 Yuanmingyuan West Road; \\ Beijing 100193; P.R. China \\ * Corresponding author. \\ phone: 1-215-233-6655; fax: 1-215-233-6642; e-mail: Yelena.Sapozhnikova@ars.usda.gov
}

Disclaimer: Mention of brand or firm names does not constitute an endorsement by the U.S. Department of Agriculture above others of a similar nature not mentioned. 


\section{Abstract}

An easy and reliable high-throughput analysis method was developed and validated for 192 diverse pesticides and 51 environmental contaminants (13 PCB congeners, 14 PAHs, 7

4 PBDE congeners, and 17 novel flame retardants) in cattle, swine, and poultry muscle. Sample preparation was based on the "quick, easy, cheap, effective, rugged and safe" (QuEChERS) approach using filter-vial dispersive solid-phase extraction (d-SPE) cleanup. Split final extracts were analyzed in parallel by low-pressure (vacuum outlet) GC-MS/MS and UHPLC-MS/MS (10 min each), providing an additional degree of confirmation for 55 overlapping LC- and GCamenable pesticides. Analyte protectants were utilized to improve sensitivity and decrease matrix effects in GC analysis, and only filtration of initial extracts was enough to avoid ion suppression in UHPLC-MS/MS. The method was validated at three spiking levels $(10,25$, and $100 \mathrm{ng} / \mathrm{g})$ at or below established tolerance levels in the sample types. Satisfactory recoveries (70-120\%) and RSDs $\leq 20 \%$ were achieved for 200 analytes. The validated method was successfully applied to the analysis of real-world incurred meat samples, further demonstrating the utility of the method for implementation in regulatory and commercial laboratories.

Keywords: pesticides, environmental contaminants, meat, method validation, analysis, LPGCMS/MS, UHPLC-MS/MS

\section{Introduction}

Worldwide meat production totaled 304 million tons in 2012, with an annual average estimated consumption of 42-76 kg per person. (Food Outlook. Biannual report on global food markets, 2014) Average meat consumption in the US consists of 55\% beef and pork, followed by

$2437 \%$ poultry. (The United States Meat Industry at a Glance) Meat and poultry are important nutritional sources of proteins, essential amino acids, and bioavailable minerals and vitamins.

26 (USDA National Nutrient Database) At the same time, many anthropogenic chemicals, particularly lipophilic pesticides and environmental contaminants, may bioaccumulate in meats resulting in human exposure during consumption. Food of animal origin was estimated to contribute $>95 \%$ of the human exposure to lipophilic anthropogenic contaminants. (Boada, Sangil, Alvarez-Leon, Hernandez-Rodriguez, Henriquez-Hernandez, Camacho, et al., 2014) Because many pesticides have been shown to cause various health effects, maximum residue 
32 levels (MRLs), called tolerances in the US, have been established to control their residues in meats and protect human health. Depending on toxicity, pesticide tolerances in meats range from 10 to 3,000 ng/g, with most set between 10-100 ng/g. (Global MRL database)

Environmental contaminants, including polychlorinated biphenyls (PCBs), polycyclic aromatic hydrocarbons (PAHs), polybrominated diphenyl ethers (PBDEs) and other flame retardants (FRs) represent another group of organic lipophilic chemicals that bioaccumulate in fatty biological tissues. Dioxin-like PCBs and some PBDE congeners are recognized by the Stockholm Convention as persistent organic pollutants (POPs) based on their high persistence, toxicity, and bio-magnification in the food chain. (The Stockholm Convention on Persistent Organic Pollutants. United Nations Environmental Progamme)

Previous reports have shown that meat and poultry are the major dietary sources of PBDEs in the human diet, although the PBDE body burden has been decreasing (Huwe \& West, 2011) since penta- and octa-PBDE congeners have been banned. Nevertheless, new organic flame retardants (organophoshorous, organochlorine, and organobromine compounds) have been introduced in the commerce to comply with fire safety regulations. These new chemicals also possess lipophilic properties, high $\log \mathrm{K}_{\mathrm{ow}}$ values, suggesting their ability to bioaccumulate in animal and human tissues and warranting their monitoring in foods.

Numerous food safety programs operate around the world to help ensure that food is safe for consumption and to reduce environmental impact. Fast, simple, efficient, and cost-effective analytical methods for pesticides and other contaminants are needed in food safety monitoring programs to provide high sample throughput and accurate results. In the USA, USDA Food Safety and Inspection Service (FSIS) conducts routine monitoring of meat (cattle, swine, poultry, sheep, goats) samples to enforce regulatory compliance. The current FSIS sample preparation method for pesticide screening is based on liquid extraction of meat samples with ethyl acetate, followed by freeze-out and solid phase extraction (SPE) clean-up to remove interfering compounds, and involves multiple evaporation steps. (US Department of Agriculture, Food Safety and Inspection Service. Screening for Pesticides by LC/MS/MS and GC/MS/MS. \#CLGPST5.06) Other commonly reported methods for extracting lipophilic pesticides and environmental contaminants from meats are based on pressurized liquid extraction (PLE) with nonpolar organic solvents (e.g. hexane, ethyl acetate, dichloromethane) usually followed by cleanup with gel-permeation chromatography (GPC) to remove lipids. (Munoz, Munoz, Pineda, 
Serrahima, \& Centrich, 2012; Wu, Bao, Zhao, Wu, Han, \& Ye, 2011) These methods take much 64 time, solvent, and labor and require specialized equipment.

We have previously developed a high-throughput analytical method for multi-class

66

68

70

72

74

76

78

80

82

84

86

88

92

90 tetrabromobenzoate (TBB), 1,2,5,6-tetrabromocyclooctane (TBCO), 1,2-dibromo-4-(1,2analysis of selected pesticides and environmental contaminants in fish and shrimp tissues based on QuEChERS extraction and dispersive solid phase extraction (d-SPE) clean-up (Sapozhnikova, 2014; Sapozhnikova \& Lehotay, 2013) combined with in-vial filtration (Han, Sapozhnikova, \& Lehotay, 2014) using low pressure (LP) gas chromatography (GC) with tandem mass spectrometry (MS/MS).

The goal of this study was to optimize and validate this approach to include FSIS-priority contaminants in FSIS-regulated foods (e.g. cattle, swine, and poultry) for transfer to FSIS regulatory field service laboratories for routine monitoring in their National Residue Program (NRP). We sought to develop and validate a simple and fast method for simultaneous determination of multi-class pesticides and diverse environmental contaminants (PCBs, PAHs, PBDEs and flame retardants) in food muscle tissues to provide better quality of results for more chemicals of concern in more samples at higher throughput, less labor, and lower costs.

\section{Materials and methods}

\subsection{Chemicals and materials}

Standards of PBDE congeners (\#28, 47, 99, 100, 153, 154, and 183), PCB congeners (\#77, 81, 105, 114, 118, 123, 126, 156, 157, 167, 170, 180, and 189), and PAHs (acenaphthene,

4 acenaphthylene, anthracene, benz(a)anthracene, benzo(a)pyrene, benzo(b)fluoranthene, benzo(g,h,i)perylene, benzo(k)fluoranthene, chrysene, dibenz(a,h)anthracene, fluoranthene,

fluorene, indeno(1,2,3-cd)pyrene, naphthalene, phenanthrene, and pyrene), flame retardants [dechlorane plus (mixture of syn- and anti-isomers), hexabromobenzene (HBB);

8 hexachlorocyclopentadienyl-dibromocyclooctane (HCDBCO), 2,2',4,4',5,5'-hexabromobiphenyl (PBB 153), pentabromoethylbenzene (PBEB), pentabromotoluene (PBT), 2-ethylhexyl-2,3,4,5dibromoethyl)cyclohexane (TBECH), tribromoneopentyl alcohol (TBNPA), and tris(1,3dichloro-2-propyl)phosphate (TDCPP)] were all purchased from AccuStandard (New Haven, CT; USA). Standards of 1,2,4,5-tetrabromo-3,6-dimethylbenzene (TBX), tris(2-chloroethyl) 
94 phosphate (TCEP), tris(1-chloro-2-propyl)phosphate (TCPP), and triphenyl phosphate (TPP) were purchased from Sigma-Aldrich (St. Louis, MO; USA), and dechlorane 604 standard was from Santa Cruz Biotechnology (Santa Cruz, CA; USA). Pesticide standards were from the Environmental Protection Agency's National Pesticide Repository (Fort Meade, MD; USA) and 98 ChemService (West Chester, PA; USA).

For use as internal standards (IS), ${ }^{13} \mathrm{C}_{12}-2,2,4,4,5,5$-hexachlorobenzene $\left({ }^{13} \mathrm{C}_{12}\right.$-PCB 153$)$, and a PAH surrogate cocktail containing acenaphthylene- $d_{8}$, benzo[a]pyrene- $d_{12}$, benzo[g,h,i]perylene- $d_{12}$, fluoranthene- $d_{10}$, naphthalene- $d_{8}$, phenanthrene- $d_{10}$ and pyrene- $d_{10}$, were purchased from Cambridge Isotope Laboratories (Andover, MA; USA). Atrazine- $d_{5}$ (ethyl$\mathrm{d} 5)$ and fenthion- $d_{6}\left(\mathrm{o}, \mathrm{o}-\right.$ dimethyl- $\left.d_{6}\right)$, were from C/D/N Isotopes (Pointe-Claire, Quebec;

104 Canada). FBDE 126 (5-fluoro-2,3,4,4,5-pentabromodiphenyl ether) and $p$-terphenyl- $d_{14}$ used as quality control (QC) standard (added just prior to injection) in GC were purchased from AccuStandard. Phenacetin-ethoxy-1- ${ }^{13} \mathrm{C}\left({ }^{13} \mathrm{C}\right.$-phenacetin) used as a QC standard for LC was acquired from Sigma-Aldrich. All standards were $\geq 98 \%$ purity.

HPLC-grade acetonitrile (MeCN) was from Fisher Scientific (Pittsburgh, PA; USA) and acetone was from Sigma-Aldrich. Deionized water of 18.2 M $\Omega$-cm was obtained using an E-Pure system from Barnstead/Thermolyne (Dubuque, IA; USA). Formic acid (88\% purity) was from Spectrum Quality Products (New Brunswick, NJ; USA). Ammonium formate $\left(\mathrm{HCO}_{2} \mathrm{NH}_{4}\right)$ was

112 from Sigma-Aldrich. Anhydrous magnesium sulfate (anh. $\mathrm{MgSO}_{4}$ ), 99.5\% purity, and primary secondary amine (PSA) were purchased from UCT (Bristol, PA; USA). C18 (40 $\mu \mathrm{m})$ was

114 purchased from Thomas Scientific (Swedesboro, NJ; USA), and zirconium-based Z-Sep sorbent was from Supelco (Bellefonte, PA; USA). Filter vials with $0.45 \mu \mathrm{m}$ polyvinylidene fluoride 116 (PVDF) filters were from Thomson Instrument Co. (Oceanside, CA; USA). Two types of filter vials were used: 1) for filter-vial d-SPE in GC, screw-cap vials with short plungers allowed room 118 for sorbent in the bottom shell receptacles; and 2) for filtering-only in LC, slit-septa snap-cap filter vials had standard length plungers. A press tray for simultaneous filtering of up to 48 filter 120 vials was provided by Thomson Instrument Co.

A working standard mixture of the environmental contaminants, containing a total of 51 122 PCBs, PBDEs, PAHs, and FRs and 192 pesticides was prepared at $2.5 \mathrm{ng} / \mu \mathrm{L}$ in MeCN/acetone, except 10-fold lower PCB concentrations $(0.25 \mathrm{ng} / \mu \mathrm{L})$. This mixture was used to prepare spiking and calibration standard solutions in MeCN. The IS solution in MeCN contained a mixture of 
atrazine- $d_{5}$ and fenthion- $d_{6}$ at $5 \mathrm{ng} / \mu \mathrm{L}, \mathrm{FBDE}$ at $2.5 \mathrm{ng} / \mu \mathrm{L}$, isotopically-labeled IS for PAHs at 1

$126 \mathrm{ng} / \mu \mathrm{L}$, and ${ }^{13} \mathrm{C}_{12}$-PCB 153 at $0.1 \mathrm{ng} / \mu \mathrm{L}$. Atrazine- $d_{5}$ was used as an IS for all pesticides, and fenthion- $d_{6}$ served as a back-up IS.

A mixture of analyte protectants (APs) contained ethylglycerol at $10 \mu \mathrm{g} / \mu \mathrm{L}$, gulonolactone and D-sorbitol each at $1 \mu \mathrm{g} / \mu \mathrm{L}$, and shikimic acid at $0.5 \mu \mathrm{g} / \mu \mathrm{L}$ was prepared in 4/1 (v/v) MeCN/water with $0.5 \%$ formic acid. Also, this solution contained the QC standard for GC of $0.438 \mathrm{ng} / \mu \mathrm{L} p$-terphenyl- $d_{14}$. A separate solution of ${ }^{13} \mathrm{C}$-phenacetin at $4 \mu \mathrm{g} / \mathrm{mL}$ was

132 prepared in MeCN for use as the QC standard in LC.

Ten $\approx 500 \mathrm{~g}$ samples each of cattle, pork, and poultry muscle tissue from different parts of the animal (grown organically), were purchased from local grocery stores. For example, chicken wings, breast, thigh, drumsticks, and whole cornish hens were used as poultry samples. All samples were filleted into skinless and boneless pieces, frozen and comminuted with dry ice using a Robot Coupe (Ridgeland, MS; USA) RSI 2Y1 chopper to produce a fine powder, which were stored in glass jars at $-18^{\circ} \mathrm{C}$ until analysis. A Glas-Col (Terre Haute, IN; USA) platform pulsed-vortexing mixer was used for extraction, (Sapozhnikova \& Lehotay, 2015) and a ThermoFisher (Waltham, MA; USA) Sorvall Legend RT centrifuge was used for centrifugation.

\subsection{Low-pressure gas chromatography tandem mass spectrometry (LPGC-MS/MS) analysis}

A separation of 211 GC-amenable compounds (199 analytes, 11 IS, and 1 QC standard) was performed on a $15 \mathrm{~m} \times 0.53 \mathrm{~mm} \times 1 \mu \mathrm{m}$ film thickness DB-5ms GC column (Agilent; Santa Clara, CA; USA) connected at the inlet to a $5 \mathrm{~m} \times 0.18 \mathrm{~mm}$ non-coated restrictor/guard column from Restek (Bellefonte, PA; USA). The connection between the two columns was secured by Ultimate union connectors (Agilent) as previously described. (Koesukwiwat, Lehotay, \&

148 Leepipatpiboon, 2011) The calculated virtual column length used for constant flow of $2 \mathrm{~mL} / \mathrm{min}$ helium carrier gas was $5.5 \mathrm{~m} \times 0.18 \mathrm{~mm}$ i.d. (vacuum outlet). The instrument was an Agilent 7890A/7000B gas chromatograph/triple quadrupole tandem mass spectrometer (the GC was upgraded to $220 \mathrm{~V}$ to allow fast temperature ramps). The GC oven temperature program started at

$15270^{\circ} \mathrm{C}$, where it was held for $1.5 \mathrm{~min}$, followed by ramps of $80^{\circ} \mathrm{C} / \mathrm{min}$ to $180^{\circ} \mathrm{C}, 40^{\circ} \mathrm{C} / \mathrm{min}$ to $250^{\circ} \mathrm{C}$, and $70^{\circ} \mathrm{C} / \mathrm{min}$ to $320^{\circ} \mathrm{C}$, where it was held for $3.4 \mathrm{~min}$, totaling $10 \mathrm{~min}$. Injection volume 154 was $2.5 \mu \mathrm{L}$ into a Multi-Mode Inlet (MMI) from Agilent operated as a programmable temperature vaporizer with solvent vent mode. The initial MMI temperature was held at $80^{\circ} \mathrm{C}$ for 
$0.31 \mathrm{~min}$ (at which point the vent was closed), then ramped to $320^{\circ} \mathrm{C}$ at $420^{\circ} \mathrm{C} / \mathrm{min}$, and held at $320^{\circ} \mathrm{C}$ until the end of the run. Rinsing solutions of water/MeCN/acetone/methanol (1/1/1/1, v/v) for Wash 1 and $\mathrm{MeCN}$ for Wash 2 were used to prevent the syringe from clogging when using sugary APs in the final extracts.

The MS transfer line was at $250^{\circ} \mathrm{C}$ and ion source was $320^{\circ} \mathrm{C}$. Electron ionization (EI) at $-70 \mathrm{eV}$ was used in MS/MS after a 2 min solvent delay; quadrupole temperatures were $150^{\circ} \mathrm{C}$, and collision gas (nitrogen) was at $1.5 \mathrm{~mL} / \mathrm{min}$ and quench gas (helium) was $2.25 \mathrm{~mL} / \mathrm{min}$. Two multiple reaction monitoring $(\mathrm{MRM})$ transitions were selected for each analyte, and the MRM method was divided into 29 time segments, each containing 62 MRM transitions with a dwell time of $2.5 \mathrm{~ms}$ and "wide" MS resolution for all transitions. In the process of method development, we observed some large interferences from matrix for some analytes, which resulted in our replacement of the original (and perhaps the most intense quantifier ions) with others not susceptible to matrix interferences. Quantifier and qualifier transitions, collision energy $(\mathrm{CE})$, and average retention times $\left(\mathrm{t}_{\mathrm{R}}\right)$ for LPGC-MS/MS analysis are presented in Table S1. MassHunter software was used for instrument control and data acquisition/processing.

\subsection{Ultrahigh-performance liquid chromatography tandem mass spectrometry (UHPLC-} MS/MS) analysis

A Waters (Milford, MA; USA) Acquity UPLC-TQD, controlled by MassLynx software was used for UHPLC-MS/MS of 98 pesticides (55 overlapping with LPGC-MS/MS). A Waters

176 BEH C18 column, $100 \mathrm{~mm} \times 2.1 \mathrm{~mm}$ i.d., $1.7 \mu \mathrm{m}$ particles, integrated with a Waters VanGuard pre-column of $5 \mathrm{~mm} \times 2.1 \mathrm{~mm}$ i.d., $1.7 \mu \mathrm{m}$ BEH C18 particles, was used for separation at $40^{\circ} \mathrm{C}$.

178 The mobile phase consisted of: (A) 95/2.5/2.5 (v/v/v) $\mathrm{H}_{2} \mathrm{O} / \mathrm{MeOH} / \mathrm{MeCN}$ and (B) 1/1 (v/v) $\mathrm{MeOH} / \mathrm{MeCN}$, both solutions containing a mixture of $20 \mathrm{mM}$ ammonium formate and $0.1 \%$ formic acid. The gradient was $95 \% \mathrm{~A}$ for $0.25 \mathrm{~min}$, then ramped to $99.8 \% \mathrm{~B}$ from $0.25 \mathrm{~min}$ to $7.75 \mathrm{~min}$, followed by $1.00 \mathrm{~min}$ hold time, after which the column was re-equilibrated for 1.45 min, making the total run time $10.25 \mathrm{~min}$. The flow rate was $0.45 \mathrm{~mL} / \mathrm{min}$, and the injection volume was $2 \mu \mathrm{L}$ (autosampler tray at $18^{\circ} \mathrm{C}$ ).

Electrospray positive ionization (ESI+) was used for the analysis of $100 \mathrm{LC}$-amenable compounds (98 pesticides, 1 IS and 1 QC standard) with 3 MRM transitions for all but a few analytes (Table S2). Fixed parameters included $2 \mathrm{kV}$ capillary voltage, $3 \mathrm{~V}$ extractor voltage, 
$120^{\circ} \mathrm{C}$ source temperature, $450^{\circ} \mathrm{C}$ desolvation temperature, $1000 \mathrm{~L} / \mathrm{hr}$ desolvation gas flow, 50

188

$\mathrm{L} / \mathrm{hr}$ cone gas flow. The gas cones were cleaned and changed between each long sequence of sample extracts.

One precursor and two product ions were selected, and identification was based on 3 ion transitions, their ratios, and $t_{R}$. MRM transitions were scheduled within \pm 0.2 min for each analyte expected $t_{R}$ with auto-dwell time automatically adjusted by the software. Table $S 2$ lists $t_{R}$, selected ions, cone voltages, and optimized CEs for LC-amenable analytes.

\subsubsection{Post-column infusion}

To evaluate d-SPE cleanup efficiency and matrix effects (MEs) in UHPLC-MS/MS analysis, a post-column infusion approach (Stahnke, Reemtsma, \& Alder, 2009) was employed for 5 selected pesticides. Blank reagents and matrix extracts with and without d-SPE cleanup were injected in the UHPLC method as usual and the different infused pesticides $(25 \mathrm{ng} / \mu \mathrm{L}, 20$ $\mu \mathrm{L} / \mathrm{min}$ ) were monitored throughout the chromatogram in MS/MS. The acquired data were subjected to smoothing with a smooth factor $=2$, window size $= \pm 5$ scans, and applying a mean smoothing method in Smooth Tool of the MassLynx software, thus resulting in 508 smoothed data points for each MRM chromatogram for each sample matrix and treatment.

\subsection{Sample preparation}

The sample preparation procedure was based on the previously reported QuEChERS approach using filter-vial d-SPE with a few modifications (Han, Sapozhnikova, \& Lehotay, 2014). For example, a sample test portion of $5 \mathrm{~g}$ rather than $10 \mathrm{~g}$ was used to save cost of reagents while still representing the bulk sample, in accordance with finding from a recent study. (Sapozhnikova \& Lehotay, 2015)

Several experiments were conducted to re-assess and optimize the method for the new matrices. For example, the use of APs was evaluated and integrated into the final LPGC-MS/MS method. Also, different d-SPE sorbents and their combinations were tested in filter-vial d-SPE to evaluate recoveries of the selected contaminants. In this experiment, $4 \mathrm{~mL} \mathrm{H}_{2} \mathrm{O}$ (reagent blank) was fortified with the analytes $100 \mathrm{ng} / \mathrm{mL}$, and extracted with $4 \mathrm{~mL}$ of MeCN using a Glas-Col shaker for 10 min at $80 \%$ setting with a maximum pulsing. Then, $2 \mathrm{~g}$ of $\mathrm{HCO}_{2} \mathrm{NH}_{4}$ was added, and the tubes were shaken for $1 \mathrm{~min}$ and centrifuged for $3 \mathrm{~min}$ at $4150 \mathrm{rpm}$ (3711 rcf). Aliquots 
$218(0.5 \mathrm{~mL})$ of the extracts were placed into $0.45 \mu \mathrm{m}$ PVDF filter-vials for d-SPE cleanup as follows (each in triplicate): (1) $75 \mathrm{mg}$ anh. $\mathrm{MgSO}_{4}$, (2) $75 \mathrm{mg}$ anh. $\mathrm{MgSO}_{4}+25 \mathrm{mg}$ of C18, (3)

$22075 \mathrm{mg}$ anh. $\mathrm{MgSO}_{4}+25 \mathrm{mg}$ of PSA, (4) $75 \mathrm{mg}$ anh. $\mathrm{MgSO}_{4}+25 \mathrm{mg}$ of Z-Sep, (5) $75 \mathrm{mg}$ anh. $\mathrm{MgSO}_{4}+25 \mathrm{mg}$ of each C18, PSA, and Z-Sep. The resulting extracts were analyzed by both

222 LPGC-MS/MS and UHPLC-MS/MS.

Based on these experiments, the final sample preparation protocol was as follows: (1) $5 \mathrm{~g}$ 224 test portion is weighed into a $50 \mathrm{~mL}$ polypropylene centrifuge tube $(3.5 \mathrm{~mL}$ water served as reagent blank); (2) $100 \mu \mathrm{L}$ of IS solution is added (or spike solution including IS), and the tubes are briefly vortexed and $15 \mathrm{~min}$ is allowed for integration into the muscle tissue; (3) $5 \mathrm{~mL}$ of $\mathrm{MeCN}$ is added using a bottle dispenser, and the samples are extracted on the platform shaker for

$22810 \mathrm{~min}$ at $80 \%$ setting with a maximum pulsation; (4) $2.5 \mathrm{~g}$ of $\mathrm{HCO}_{2} \mathrm{NH}_{4}$ is added to induce phase separation, and the tubes are shaken as before for $1 \mathrm{~min}$; and (5) the tubes are centrifuged 230 at room temperature for $3 \mathrm{~min}$ at $4150 \mathrm{rpm}$ (3711 rcf).

For UHPLC-MS/MS, $0.5 \mathrm{~mL}$ of this extract, $25 \mu \mathrm{L}$ of QC standard ${ }^{13} \mathrm{C}$-phenacetin, and $71.4 \mu \mathrm{L}$ of $\mathrm{MeCN}$ (to compensate for calibration standard volumes) are transferred to the shell portion of the filter vial and filtered through $0.45 \mu \mathrm{m}$ PVDF (without d-SPE) by pressing the 234 plunger portion of the vial into its shell.

For LPGC-MS/MS analysis, filter-vial d-SPE cleanup is performed as follows: (A) transfer $0.6 \mathrm{~mL}$ of extracts into a vial shells containing a $180 \mathrm{mg}$ sorbent mixtures consisting of $90 \mathrm{mg}$ anh. $\mathrm{MgSO}_{4}+30 \mathrm{mg}$ each of PSA, C18, and Z-Sep; (B) place the plungers halfway into

238 the shells in a tray, and shake the tray vigorously for $30 \mathrm{~s}$; (C) use the press to fully depress the plungers to filter the extracts; (D) transfer $175 \mu \mathrm{L}$ of the cleaned/filtered extracts to an amberglass autosampler vial containing a micro-vial insert, plus $40 \mu \mathrm{L}$ of the AP mixture containing $p$ terphenyl- $d_{14}$ and $25 \mu \mathrm{L}$ of MeCN to match sample volumes with the calibration standards; (E) shake the vials end-to-end for $10 \mathrm{~s}$ (this is important because diffusion is initially slow).

\subsection{Method validation}

The three days validation procedure involved analysis of beef (day 1), chicken (day 2) and pork (day 3) samples. On each day, 4 replicate test portions of the 10 different comminuted beef/chicken/pork samples were weighed into $50 \mathrm{~mL}$ tubes. One portion was a matrix blank, and the others were spiked at 10, 25, and $100 \mathrm{ng} / \mathrm{g}$, yielding 10 replicates for each matrix type at each 
level. Equal parts of the 10 muscles of each kind of meat were mixed together to make an

250 "average" blank matrix, extracts of which were used in matrix-matched (MM) calibration standards at $0,5,10,25,100$, and $200 \mathrm{ng} / \mathrm{g}$ (10 times lower for PCBs). Also, a reagent blank was

252 included each day during the validation study. Reagent-only (RO) calibration standards were prepared in $\mathrm{MeCN}$ at the same concentrations as MM standards.

\section{Results and Discussion}

\subsection{Instrumental method}

Diverse pesticides were selected to represent different classes of non-polar and relatively 258 polar pesticides (stable organochlorine, organophosphorous, pyrethroids, triazines, carbamates, strobins, azoles, urea, etc.) totaling to 192 compounds. Among environmental contaminants, 14

260 PAHs were selected representing the US EPA priority PAHs, including carcinogenic ones; 13 PCB congeners identified by the World Health Organization (WHO), including dioxin-like

262 congeners; 7 common PBDE congeners, and 17 flame retardants representing large volume production chemicals used to replace the banned PBDE flame retardants. Fast LPGC and

264 UHPLC were used in parallel (10 min each) for compounds separation and tandem triple quadrupole mass spectrometry was used in both cases. Fifty-five pesticides were both GC- and

266 LC-amenable allowing an additional degree of confirmation with an orthogonal technique.

\subsection{Sample preparation}

\subsubsection{Comparison of different sorbents in filter-vial d-SPE approach}

The most common d-SPE sorbents used to remove interfering lipids and fats from extracts are C18, PSA (Hou, Han, Dai, Yang, \& Yi, 2013), and a recently introduced zirconium

272 dioxide based sorbent called Z-Sep. We have previously reported successful use of these sorbents for cleaning fish extracts. In order to investigate retention properties of these sorbents

274 for the selected 243 analytes, we conducted a recovery experiment as described in section 2.4. The recoveries of 243 analytes were calculated $v s$. representative IS using RO calibration curves.

276 Table S3 and Table S4 list calculated recoveries and standard deviations (SDs) of the analytes with different d-SPE sorbents measured by LPGC-MS/MS and UHPLC-MS/MS, respectively. recoveries above $70 \%$. However, some analyte adsorption was observed for 16 pesticides 
resulting in decreased recoveries as presented in Figure S1. Specifically, C18 retained trinexapac-ethyl (46\% recovery), and spinosyn A and D isomers (38 and 37\%, respectively), and PSA retained trinexapac-ethyl (20\% recovery), flumetsulam and chlorsulfuron (6 and $8 \%$ recoveries, respectively). Imazaquin and imazethapyr were completely retained by all tested sorbents except for C18. Z-Sep retained 14 tested analytes (Figure S1): imazaquin, imazethapyr, and trinexapac ethyl (all 0\% recovery), methamidophos (7-11\%), cyromazine (30\%), spinosyns A\&D, oxydemethon-methyl, chlorpyrifos oxon (all 40\% recoveries), acephate, flutriafol, omethoate, paraoxon-methyl (50\%), and dichrotophos (60\%). Our results for acephate, omethoate, methamidophos and dichrotophos recoveries with Z-Sep were practically identical to the results of a recent study by Morris and Schriner (Morris \& Schriner, 2015) reporting pesticide recoveries in avocado after automated column SPE (c-SPE) cleanup with Z-Sep. Interestingly, the latter study used $30 \%$ more Z-Sep per $\mathrm{mL}$ of avocado extract compared to our study which used reagent blank samples (no matrix), and different cleanup format (c-SPE vs. filter-vial d-SPE). Another recent study (Rajski, Lozano, Ucles, Ferrer, \& Fernandez-Alba, 2013) reported decreased recovery of cyromazine (50\%) with Z-Sep d-SPE cleanup for avocado extracts, similar to our finding, where cyromazine recovery was only $30 \%$. In this study, the amount of Z-Sep used per mL of extract was $30 \%$ less than in our study. Also, this study reported 80-100\% recovery for methamidophos, flutriafol, and dichrotophos, which differed from our results (11-60\%). Perhaps, decreased amount of Z-Sep sorbent per mL of extract in the latter study explains greater recoveries of these pesticides.

\subsubsection{Matrix effects (MEs) in LPGC-MS/MS}

Meat samples are very complex matrices, containing proteins, lipids, saturated and monounsaturated fatty acids (USDA National Nutrient Database) and even thorough cleaning of the extracts does not prevent matrix co-extractives in the final extracts. In order to estimate MEs caused by matrix components in the injected extracts, calibration standard solutions at $0,5,10$, 50, 100, and $200 \mathrm{ng} / \mathrm{g}$ were prepared in $\mathrm{MeCN}(\mathrm{RO})$ and matrix-matched (MM) extracts. A mixture of muscle tissue samples containing equal parts of beef, chicken, and pork (by weight) was extracted and subjected to d-SPE cleanup as described above, and the aliquots of this extract were used to prepare MM calibration standards. 
In LPGC-MS/MS, comparison of the sensitivities (slopes) for MeCN and MM calibration curves for environmental contaminants (PBDEs, PCBs, PAHs and FRs) and 69 pesticides

312 showed very little differences, demonstrating low $(\leq 20 \%)$ MEs for these analytes (Figure S2). However, for the vast majority of pesticides, analyte responses were greater in MM calibration

314 compared to MeCN-only (Figure S2), demonstrating matrix-induced response enhancement previously described (Erney, Gillespie, Gilvydis, \& Poole, 1993; Erney, Pawlowski, \& Poole, 1997) Indeed, hydrophilic compounds containing relatively polar functional groups are prone to interact with active sites of the inlet liner, column, and ion source when injected in RO solutions without matrix. When MM calibration is used, matrix components fill the active sites, causing increase in analyte responses compared to RO calibration.

AP solution was added to the final LPGC-MS/MS extracts before injection as described in section 2.4. Addition of APs had no effect on sensitivities of PBDEs, PCBs, PAHs, FRs and 69 pesticides that demonstrated $\leq 20 \%$ MEs in MeCN vs. MM. However, APs significantly increased the signals of other pesticides, which had already exhibited higher MEs. As presented in Figure S2, the most polar and problematic pesticides gave greatly increased analyte responses upon the addition of APs, reducing loss of polar analytes on active sites. For example, acephate, propargite, and pyraclostrobin responses were 9-10 times greater after APs addition to $\mathrm{MeCN}$ calibration standards (Figure S2). Nevertheless, polar pesticides prone to adsorption on active sites of inlet liner, column, and ion source exhibited rather different behaviors. Figure S2 lists pesticides in order of retention times, which shows that earlier eluting analytes present a different pattern compared to late eluting analytes that give much greater responses in MM + APs solutions than in MeCN+APs. A similar phenomenon was reported previously indicating that APs could not fully compensate for MEs of those late-eluting pesticides (Mastovska, Lehotay, \& Anastassiades, 2005). Therefore, we chose to use MM calibration standards with addition of APs for LPGC-MS/MS analysis.

In addition to APs leading to increased sensitivity, improved peak shapes and decreased peak tailing were observed in many cases. As exhibited in Figure S3, significant peak shape improvements were observed with introduction of APs, resulting in sharper, narrower, taller peaks, as well as reduced peak tailing for susceptible analytes. 
Post-column infusion of 5 selected pesticides with different physicochemical properties

342 was performed to evaluate effectiveness of d-SPE cleanup via measuring MEs. It has been reported that thorough cleanup of complex biological extracts reduces amounts of co-extractives

344 and helps to decrease MEs. Because our results showed that some relatively polar pesticides were retained by the sorbents employed in d-SPE cleanup (C18, PSA, and Z-Sep), that works well for GC-MS/MS analysis, we aimed to determine if the cleanup is indeed beneficial for LCMS/MS analysis, too, which is generally more rugged. Our intention was to compare if ME profiles of RO and meat extracts after d-SPE cleanup were significantly different from the extracts without cleanup at all.

As reported by Stahnke et al. (Stahnke, Reemtsma, \& Alder, 2009), MEs for pesticides in ESI+ are matrix-dependent (not analyte-dependent) with analytes exhibiting similar behavior independent of their properties. Similar findings were reported for veterinary drugs by Geis et al. (Geis-Asteggiante, Lehotay, Lightfield, Dutko, Ng, \& Bluhm, 2012).

Figure S4 illustrates ME profiles of cyromazine, acetamiprid, paraoxon-ethyl and trifloxystrobin. The ME profile was also determined for fenazaquin, which was identical to trifloxystrobin's. Interestingly, only $\sim 10 \%$ differences in MEs were observed for these pesticides with d-SPE cleanup vs. samples without cleanup. Based on these results, we decided not to use d-SPE cleanup for extracts subjected to LC-MS/MS analysis. A recent study analyzing pesticides in cocoa beans utilized the same approach where d-SPE was employed for GC extracts, and no clean-up was used for LC-MS/MS analysis. (Zainudin, Salleh, Mohamed, Yap, \& Muhamad, 2015)

Similarly, the signal difference between RO, pork, chicken, and beef matrices (both with and without cleanup) was also only $\sim 10 \%$ for 4 pesticides, and $\sim 20 \%$ for paraoxon-ethyl. These

364 findings are similar to the results of Stahnke et al. who reported that $80 \%$ out of 129 pesticides had a ME difference $<10 \%$ for simple matrices like pears, and up to $26 \%$ differences for more difficult matrices like orange (Stahnke, Reemtsma, \& Alder, 2009).

\subsection{Method validation}

\subsubsection{Linearity, sensitivity and matrix effects (MEs)}

In LPGC analysis, visual inspection of calibration curves (RO and MM) consisting of 6 points at $0,5,10,25,100$, and $200 \mathrm{ng} / \mathrm{g}$ (10 times lower for PCBs) for each matrix demonstrated 
372 linear regression coefficient values of $\left(R^{2}\right) \geq 0.99$. Lowest calibration levels (LCLs) for the majority of analytes were $5 \mathrm{ng} / \mathrm{g}(0.5 \mathrm{ng} / \mathrm{g}$ for PCBs), with exceptions of atrazine, atrazine-

374 desethyl, dieldrin, endrin, endrin ketone, ethoprop, fenpropathrin, HCDBCO, hexazinone, cisand trans-nonaclor, PBB 153, PBDE 153\&154, PBEB, prochloraz, TDCPP, thiamethoxam

$376(\mathrm{LCL}=10 \mathrm{ng} / \mathrm{g}$ ), azoxystrobin, dechlorane 604, $\alpha$ - and $\beta$-endosulfan, endosulfan sulfate, PBDE 183, TBB (LCL=25 ng/g), and HBB (LCL=100 ng/g) (See Table S1).

For UHPLC analysis, LCLs were $\leq 5 \mathrm{ng} / \mathrm{g}$ for all pesticides (Table S2), except for ethofumesate, hexythiazox, and paraoxon-methyl (10 ng/g); diflubenzuron, novaluron, resmethrin, and tolylfluanid (25 ng/g); and bifenthrin, $\tau$-fluvalinate $(100 \mathrm{ng} / \mathrm{g})$, and permethrin (200 ng/g). We attempted to include pyrethroid pesticides (bifenthrin, cypermethrin,

382 deltamethrin, $\tau$-fluvalinate, resmethrin, and permethrin) in UHPLC-MS/MS analysis as previously reported by Mastovska et al. (Mastovska, Dorweiler, Lehotay, Wegscheid, \& Szpylka, 2010). Different adducts $\left([\mathrm{M}+\mathrm{H}]^{+},\left[\mathrm{M}+\mathrm{NH}_{4}\right]^{+}\right.$, and $\left.[\mathrm{M}+\mathrm{Na}]^{+}\right)$were tested and compared in MS/MS experiments, and the most intense transitions were selected (see table S2). However, under the selected UHPLC-MS/MS conditions, the sensitivities of the selected pyrethroids was very low compared to their sensitivities in LPGC-MS/MS. Two other recent studies also reported greater LOQs for pyrethroid pesticides when analyzed by LC compared to the most of LCamenable pesticides (Jabot, Fieu, Giroud, Bulete, Casabianca, \& Vulliet, 2015; Kasiotis, Anagnostopoulos, Anastasiadou, \& Machera, 2014). Nevertheless, this trial shows a potential for analysis of some pyrethroids by LC-MS/MS, albeit at much higher concentrations.

In terms of linearity of MM calibrations with UHPLC, all but one compound (diflubenzuron, $\mathrm{R}^{2}=0.98$ ) had linear regression coefficients $\mathrm{R}^{2} \geq 0.99$ normalized to IS atrazine$d_{5}$.

Figure S5 demonstrates the difference between MEs in GC and LC analyses. Only a few analytes fall outside of the $\pm 20 \%$ MEs box for LC-amenable compounds, despite using no cleanup. Co-extractive materials from meat samples do not contribute to MEs with ESI, and the results are rather homogenous, independent of the matrix. Since most LC-amenable pesticides are rather polar, with low $\log \mathrm{K}_{\mathrm{ow}}$ values, lipid co-extractives are not influencing their signal in ESI+ compared to EI in GC analysis. An interesting example of comparing MEs in GC and LC is omethoate that was analyzed by both techniques, and had MEs up to (-50\%) in pork in GC vs. $(-10 \%)$ in LC. 
Conversely, the majority of GC-amenable analytes demonstrated rather high MEs (up to $404100 \%$ ) with a clear differences in MEs among different meat types. MEs in pork matrix were the lowest $( \pm 20 \%)$, and MEs for the majority of analytes in chicken and beef were $20-60 \%$. Pork was the leanest matrix with 2-3 times lower fat content compared to chicken and beef, leading to practically non-existent MEs. This finding highlights the importance of MM calibrations in analysis of complex matrices to achieve accurate results.

\subsubsection{Recoveries and reproducibilities}

Method validation was conducted according to the FSIS protocol to evaluate recoveries and relative standard deviations (RSDs) of 243 analytes from pork, beef, and chicken samples ( $\mathrm{n}=10$ each) fortified at low (10 ng/g), medium (25 ng/g), and high (100 ng/g) levels. Overall, satisfactory recoveries (70-120\%) and RSD $\leq 20 \%$ were achieved for 200 analytes (82\%) (see Figure 1). The recoveries were consistent across the spiking levels, but as expected, more analytes (91-95\%) had RSDs $\leq 20 \%$ for $100 \mathrm{ng} / \mathrm{g}$ spiking levels compared to $72-80 \%$ of analytes with RSD $\leq 20 \%$ at the low $10 \mathrm{ng} / \mathrm{g}$ spiking level (10 and $1 \mathrm{ng} / \mathrm{g}$ levels for PCBs, respectively).

Most of the analytes with $<70 \%$ recoveries were either retained by sorbents in d-SPE (see section 3.2.1) or their peak areas were not normalized to an IS. For example, tri-BDE (\#28) and tetra-BDE (\#47) and tetra- (\#77 and 81), and penta-chlorobiphenyl (CB) congeners (\#105, 114, 118,123 , and 126 ) showed $\approx 200 \%$ recoveries when normalized to IS originally selected for PBDEs: FBDE 126 (penta-BDE) and PCBs: ${ }^{13} \mathrm{C}_{12}$-PCB 153 (hexachlorobiphenyl). These IS had $\approx 50 \%$ recoveries through the sample preparation process, resulting in inflated recoveries for low

424 halogenated PBDE and PCB congeners. Otherwise, both IS worked well for higher brominated PBDE (penta-, hexa- and hepta-brominated congeners (\#99, 100, 153, 154 and 183) and higher

426 chlorinated PCB congeners: hexa- (\#156, 157, 167, 169), and heptachlorobiphenyl (\#170, 180, and 189), exhibiting similar behavior through the sample preparation process as a result of 428 similar chemical structure and properties (in particular, $\log \mathrm{K}_{\mathrm{ow}}$ values). This emphasizes the importance of careful method validation and continuous QA/QC along each processed batch of samples, as the fact of inflated recoveries is obvious in the case of spiked samples, but may cause inaccurate results in the case of unknown concentrations.

In our previous studies using FBDE 126 and ${ }^{13} \mathrm{C}_{12}$-PCB as IS for PCBs and PBDEs in fish and shrimp tissues (Han, Sapozhnikova, \& Lehotay, 2014; Sapozhnikova \& Lehotay, 2013), 
434 the recoveries were satisfactory for all of the PBDE and PCB congeners, indicating matrixdependent behavior of these IS. It is commonly known, that fish have higher concentrations of polyunsaturated fatty acids compared to meats, which contain more saturated fatty acids that interact with lipophilic PCBs and PBDEs in a different manner. Huwe and West (2011) conducted a study measuring PBDEs in meat and poultry utilizing $10{ }^{13} \mathrm{C}$-labeled PBDE internal standards for quantification of 16 native PBDEs to cover the range of their properties (Huwe \& West, 2011). Another recent study performed quantification of tri- and penta-BDEs using fluorinated BDE 69 (FBDE 69) and hexa- and nona-BDEs using FBDE 160 (Labunska, Harrad,

442 Wang, Santillo, \& Johnston, 2014). In the absence of a suitable IS for low halogenated PCBs and PBDEs, we resorted to calculating their "true" recoveries without normalizing to IS, which led to $444<70 \%$ recoveries.

Recoveries of environmental contaminants and GC-amenable pesticides are presented in

446 Figure 2. Seventeen novel FR compounds in this study represented a diverse group of chlorinated, brominated, and organophosphate chemicals. For the majority of FRs, the recoveries and RSDs were satisfactory, however, a few brominated FRs (PBEB, PBT, TBX, and HBB) had low recoveries (26-63\%). Dechlorane 604 and HBB had low sensitivity, and were only measurable at $100 \mathrm{ng} / \mathrm{g}$ spiking levels, with dechlorane 604 recoveries ranging from 82 to $103 \%$ and $\mathrm{HBB}$ recoveries reaching $43-63 \%$ at this spiking level.

Some pesticides showed a different pattern in recoveries depending on the meat type. For example, propachlor had relatively low recoveries (14-46\%) in fortified beef samples compared 454 to chicken (99-102\%) and pork samples (89-96\%). HCB's recoveries were lower in pork samples (46-56\%) vs. recoveries in beef (78-112\%) and chicken (67-86\%).

Among 55 pesticides analyzed by both LC and GC techniques, the majority of compounds had similar recoveries. However, because some pesticides have been shown to 458 adsorb on Z-Sep used in cleanup prior to GC, their recoveries were greater when analyzed by LC-MS/MS without d-SPE cleanup. These included acephate (11-49\% recoveries in GC vs. 46-

$46054 \%$ in LC), methamidophos (1-30\% GC vs. $37-46 \%$ in LC), flutriafol (24-44\% in GC vs. 92$128 \%$ in LC), omethoate (17-32\% in GC vs. 60-80\% in LC) and dicrotophos (40-101\% in GC vs. $90-111 \%$ in LC).

In beef samples, $85 \%$ and $88 \%$ of analytes analyzed by GC and LC techniques, 464 respectively, had 70-120\% recoveries, and 2\% of LC-amenable analytes had recoveries $>120 \%$. 
For chicken, 89\% (GC) and 87\% (LC) of the analytes were recovered at 70-120\%, and 1\% (GC)

466 and $6 \%$ (LC) of the analytes' recoveries were calculated to be $>120 \%$. During the last day of the validation study, recoveries from pork samples were $70-120 \%$ for $75 \%$ of GC-amenable and $68 \%$ of LC-amenable analytes, with $13 \%$ and $25 \%$ of the analytes' recoveries over $120 \%$ for GC and LC analyses, respectively. The trend of increasing recoveries was observed as the validation progressed, most clearly seen in the case of LC-amenable compounds (Figure 1). We hypothesize that the solvent evaporated in the spiking solution from day 1 to day 3 , introducing a

472 bias into calculated recoveries.

\subsection{Application of the method for analysis of meat samples from the market}

The validated method was applied to determine pesticides and environmental contaminants in nine different species of meat samples supplied by USDA FSIS, including samples of beef (1), chicken (1), goat (1), lamb (1), horse (1), pork (2), and catfish (2) muscles.

478 Each sample was prepared and analyzed in triplicate, and reagent blank and matrix spike samples for chicken, pork, beef, and catfish were used for quality control.

Residues of tetraconazole $(5.7 \mathrm{ng} / \mathrm{g}), \alpha-\mathrm{BHC}(1.5 \mathrm{ng} / \mathrm{g})$, and $p, p^{\prime}-\mathrm{DDE}(0.8 \mathrm{ng} / \mathrm{g})$ were measured in the lamb sample (Table 3). Both $\alpha$-BHC and $p, p$ '-DDE are stable organochlorine pesticides that have been banned for decades, however, due to their persistence, their residues are still detected. Tetraconazole is a fungicide with an established tolerance of $10 \mathrm{ng} / \mathrm{g}$. DDT pesticides were also measured in one of the catfish samples: $p, p^{\prime}$-DDT (0.9 ng/g), $o, p^{\prime}$-DDD (1.1 $\mathrm{ng} / \mathrm{g}), o, p$ '-DDT + p,p'-DDD (9.3 ng/g), and p,p'-DDE (24 ng/g). DDT's reported half-life is approximately 30 years; it degrades into DDD and DDE. This explains the higher DDE amount measured in the catfish, and points to an old source of contamination. We have also previously reported measured DDT residues in catfish samples from the market (Sapozhnikova, 2014). A residual amount of an herbicide, metolachlor $(0.7 \mathrm{ng} / \mathrm{g})$, was also measured in the catfish, which was significantly below the tolerance $(20 \mathrm{ng} / \mathrm{g})$. Piperonyl butoxide(PBO) was also determined in one of the pork samples at $49 \mathrm{ng} / \mathrm{g}$, which was below the established tolerance of $100 \mathrm{ng} / \mathrm{g}$.

492 PBO is a synergist added to pyrethroid pesticide formulations to increase their potency. 
A multi-class, multiresidue method for analysis of 243 pesticides and environmental 496 contaminants (PCBs, PAHs, PBDEs, and FRs) was developed and validated in swine, cattle, and poultry muscle. The method is based on QuEChERS sample preparation with filter-vial d-SPE

498 prior to LPGC-MS/MS and filtration-only for UHPLC-MS/MS of 10 min analyses each. APs were used in GC analysis to improve peak shapes, sensitivities, and reduce relative MEs. The method was validated at three spiking levels (10, 25, and $100 \mathrm{ng} / \mathrm{g})$. Satisfactory recoveries (70$120 \%)$ and precision (RSDs $\leq 20 \%)$ were demonstrated for $200(82 \%)$ of the targeted analytes.

502 LCLs were $\leq 5 \mathrm{ng} / \mathrm{g}$ for $90 \%$ of the analytes allowing their determination at or below established tolerance levels. The final method was simple, fast, and inexpensive, and the overlapping 504 UHPLC-MS/MS and LPGC-MS/MS coverage of 55 pesticides allowed confirmation of the analytes in the extracts using orthogonal techniques. The validated method was used for analysis of 9 incurred meat samples from the market.

\section{Acknowledgments}

Technical assistance of Alan Lightfield, Tawana Simons, and Robyn Moten are greatly 510 appreciated. Lijun Han's sabbatical funding was provided by the China Scholarship Council. We thank Sam Ellis of Thomson Scientific for providing a press tray apparatus. Thanks to Catalina 512 Yee of USDA-FSIS for providing incurred meat samples. 


\section{References:}

Boada, L. D., Sangil, M., Alvarez-Leon, E. E., Hernandez-Rodriguez, G., Henriquez-Hernandez, L. A., Camacho, M., Zumbado, M., Serra-Majem, L., \& Luzardo, O. P. (2014). Consumption of foods of animal origin as determinant of contamination by organochlorine pesticides and polychlorobiphenyls: Results from a population-based study in Spain Chemosphere, 114, 121-128.

Erney, D. R., Gillespie, A. M., Gilvydis, D. M., \& Poole, C. F. (1993). Explanation of the matrix-induced chromatographic response enhancement of organophosphorus pesticides during open-tubular column gas-chromatography with splitless or hot on-column injection and flame photometric detection J. Chromatogr., 638(1), 57-63.

Erney, D. R., Pawlowski, T. M., \& Poole, C. F. (1997). Matrix-induced peak enhancement of pesticides in gas chromatography: Is there a solution? HRC J. High Resolut. Chromatogr., 20(7), 375-378.

Food Outlook. Biannual report on global food markets. (2014). Available at: http://www.fao.org/3/a-i4136e.pdf

Geis-Asteggiante, L., Lehotay, S. J., Lightfield, A. R., Dutko, T., Ng, C., \& Bluhm, L. (2012). Ruggedness testing and validation of a practical analytical method for $>100$ veterinary drug residues in bovine muscle by ultrahigh performance liquid chromatography-tandem mass spectrometry J. Chromatogr. A, 1258, 43-54.

Global MRL database. Available at: https://www.globalmrl.com

Han, L., Sapozhnikova, Y., \& Lehotay, S. J. (2014). Streamlined sample cleanup using combined dispersive solid-phase extraction and in-vial filtration for analysis of pesticides and environmental pollutants in shrimp Anal. Chim. Acta, 827(0), 40-46.

Hou, X., Han, M., Dai, X. H., Yang, X. F., \& Yi, S. G. (2013). A multi-residue method for the determination of 124 pesticides in rice by modified QuEChERS extraction and gas chromatography-tandem mass spectrometry Food Chem., 138(2-3), 1198-1205.

Huwe, J. K., \& West, M. (2011). Polybrominated diphenyl ethers in US meat and poultry from two statistically designed surveys showing trends and levels from 2002 to 2008 J. Agric. Food. Chem., 59(10), 5428-5434.

Jabot, C., Fieu, M., Giroud, B., Bulete, A., Casabianca, H., \& Vulliet, E. (2015). Trace-level determination of pyrethroid, neonicotinoid and carboxamide pesticides in beeswax using dispersive solid-phase extraction followed by ultra-high-performance liquid chromatography-tandem mass spectrometry Int. J. Environ. Anal. Chem., 95(3), 240-257.

Kasiotis, K. M., Anagnostopoulos, C., Anastasiadou, P., \& Machera, K. (2014). Pesticide residues in honeybees, honey and bee pollen by LC-MS/MS screening: Reported death incidents in honeybees Sci. Total Environ., 485-486, 633-642.

Koesukwiwat, U., Lehotay, S. J., \& Leepipatpiboon, N. (2011). Fast, low-pressure gas chromatography triple quadrupole tandem mass spectrometry for analysis of 150 pesticide residues in fruits and vegetables J. Chromatogr. A, 1218(39), 7039-7050.

Labunska, I., Harrad, S., Wang, M. J., Santillo, D., \& Johnston, P. (2014). Human dietary exposure to PBDEs around e-Waste recycling sites in Eastern China Environ. Sci. Technol., 48(10), 5555-5564.

Mastovska, K., Dorweiler, K. J., Lehotay, S. J., Wegscheid, J. S., \& Szpylka, K. A. (2010). Pesticide multiresidue analysis in cereal grains using modified QuEChERS method 
combined with automated direct sample introduction GC-TOFMS and UPLC-MS/MS techniques J. Agric. Food. Chem., 58(10), 5959-5972.

Mastovska, K., Lehotay, S. J., \& Anastassiades, M. (2005). Combination of analyte protectants to overcome matrix effects in routine GC analysis of pesticide residues in food matrixes Anal. Chem., 77(24), 8129-8137.

Morris, B. D., \& Schriner, R. B. (2015). Development of an automated column solid-phase extraction cleanup of QuEChERS extracts, using a zirconia-based sorbent, for pesticide residue analyses by LC-MS/MS J. Agric. Food. Chem., 63(21), 5107-5119.

Munoz, E., Munoz, G., Pineda, L., Serrahima, E., \& Centrich, F. (2012). Multiresidue method for pesticide residue analysis in food of animal and plant origin based on GC or LC and MS or MS/MS J. AOAC Int., 95(6), 1777-1796.

Rajski, L., Lozano, A., Ucles, A., Ferrer, C., \& Fernandez-Alba, A. R. (2013). Determination of pesticide residues in high oil vegetal commodities by using various multi-residue methods and clean-ups followed by liquid chromatography tandem mass spectrometry $J$. Chromatogr. A, 1304, 109-120.

Sapozhnikova, Y. (2014). Evaluation of low-pressure gas chromatography-tandem mass spectrometry method for the analysis of $>140$ pesticides in fish J. Agric. Food. Chem., 62(17), 3684-3689.

Sapozhnikova, Y., \& Lehotay, S. J. (2013). Multi-class, multi-residue analysis of pesticides, polychlorinated biphenyls, polycyclic aromatic hydrocarbons, polybrominated diphenyl ethers and novel flame retardants in fish using fast, low-pressure gas chromatographytandem mass spectrometry Anal. Chim. Acta, 758(0), 80-92.

Sapozhnikova, Y., \& Lehotay, S. J. (2015). Evaluation of different parameters in the extraction of incurred pesticides and environmental contaminants in fish J. Agric. Food. Chem., 63(21), 5163-5168.

Stahnke, H., Reemtsma, T., \& Alder, L. (2009). Compensation of matrix effects by postcolumn infusion of a monitor substance in multiresidue analysis with LC-MS/MS Anal. Chem., 81(6), 2185-2192.

The Stockholm Convention on Persistent Organic Pollutants. United Nations Environmental Progamme. Available at: http://chm.pops.int/Convention/tabid/54/Default.aspx

The United States Meat Industry at a Glance. Availavle at: https://www.meatinstitute.org

US Department of Agriculture, Food Safety and Inspection Service. Screening for Pesticides by LC/MS/MS and GC/MS/MS. \# CLG-PST5.06 Available at: www.fsis.usda.gov/shared/PDF/CLG-PST5.pdf.

USDA National Nutrient Database. Available at: http://ndb.nal.usda.gov/ndb/

Wu, G., Bao, X. X., Zhao, S. H., Wu, J. J., Han, A. L., \& Ye, Q. F. (2011). Analysis of multipesticide residues in the foods of animal origin by GC-MS coupled with accelerated solvent extraction and gel permeation chromatography cleanup Food Chem., 126(2), 646654.

Zainudin, B. H., Salleh, S., Mohamed, R., Yap, K. C., \& Muhamad, H. (2015). Development, validation and determination of multiclass pesticide residues in cocoa beans using gas chromatography and liquid chromatography tandem mass spectrometry Food Chem., 172, 585-595. 
Figure 1. Recoveries and relative standard deviations (RSDs) of 243 analytes using LPGCMS/MS and UHPLC-MS/MS analysis

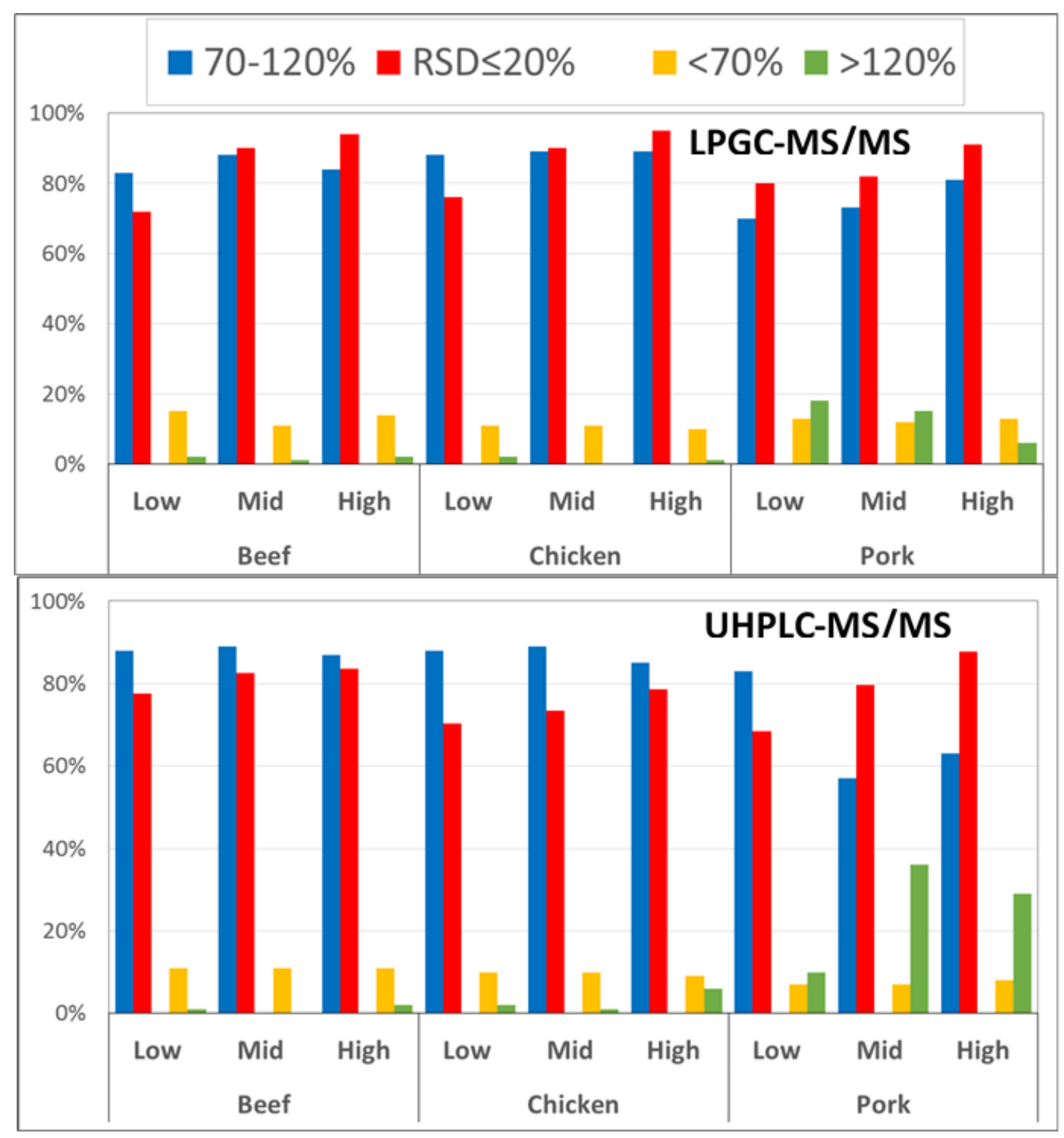


Figure 2. Average recoveries of environmental contaminants (PCBs, PBDEs, PAHs and flame retardants (FRs)) and pesticides from meat samples fortified at 10 (low), 25 (mid) and $100 \mathrm{ng} / \mathrm{g}$ (high) by LPGC-MS/MS. Error bars represent standard deviations ( $\mathrm{n}=10$ samples).

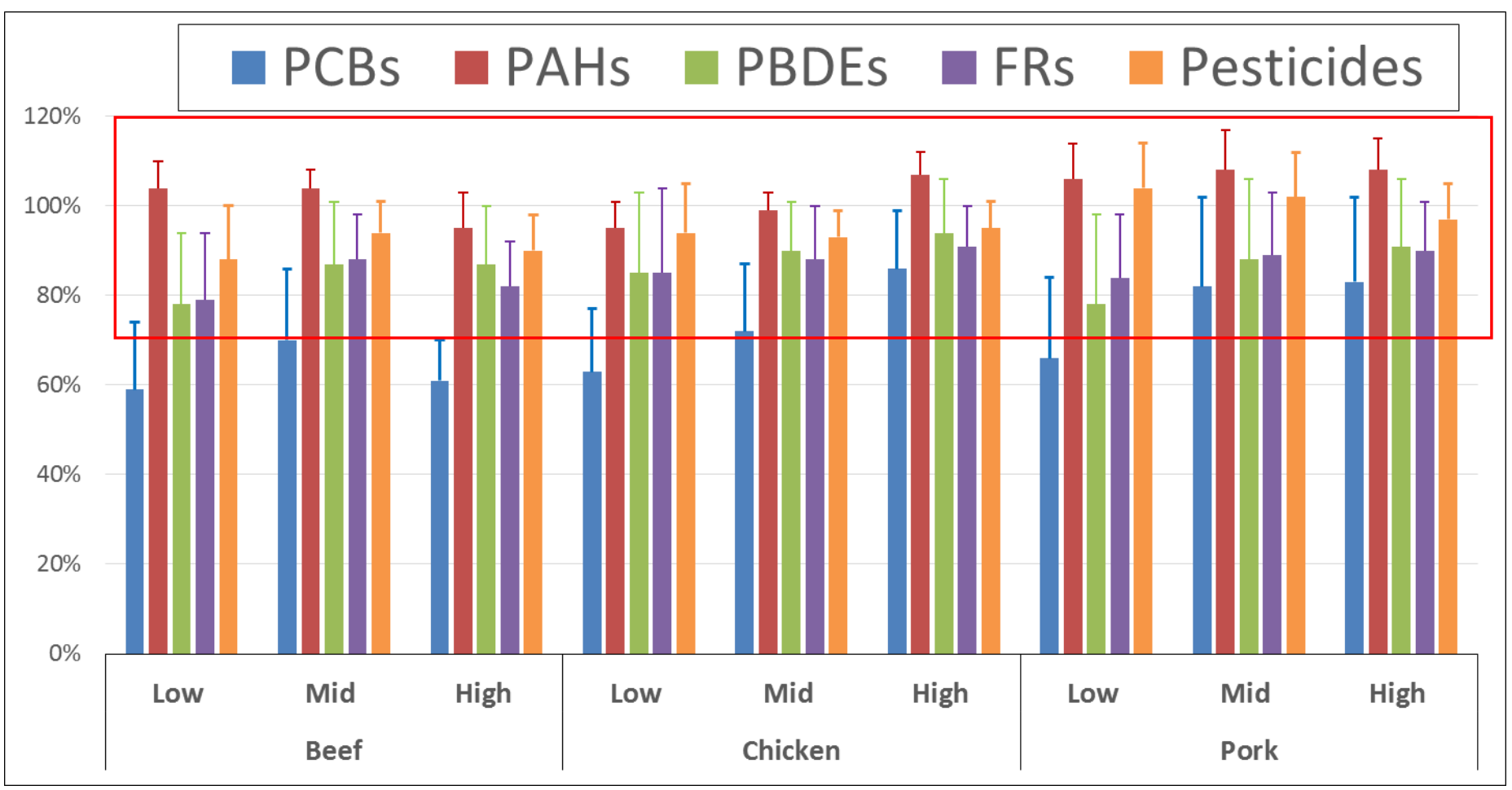


Table 1. Average \%recoveries \pm standard deviation (SD), $n=10$ each level and matrix, in method validation of 199 pesticides and environmental contaminants in cattle, pork, and chicken muscle tissues at three spiking levels using LPGC-MS/MS for analysis.

\begin{tabular}{|c|c|c|c|c|c|c|c|c|c|}
\hline \multirow[b]{2}{*}{ Analyte } & \multicolumn{3}{|c|}{ CATTLE } & \multicolumn{3}{|c|}{ CHICKEN } & \multicolumn{3}{|c|}{ PORK } \\
\hline & $10 \mathrm{ng} / \mathrm{g}$ & $25 \mathrm{ng} / \mathrm{g}$ & $100 \mathrm{ng} / \mathrm{g}$ & $10 \mathrm{ng} / \mathrm{g}$ & $25 \mathrm{ng} / \mathrm{g}$ & $100 \mathrm{ng} / \mathrm{g}$ & $10 \mathrm{ng} / \mathrm{g}$ & $25 \mathrm{ng} / \mathrm{g}$ & $100 \mathrm{ng} / \mathrm{g}$ \\
\hline acenaphthene & $102 \pm 6$ & $100 \pm 6$ & $90 \pm 8$ & $95 \pm 7$ & $96 \pm 4$ & $103 \pm 3$ & $109 \pm 8$ & $106 \pm 3$ & $103 \pm 6$ \\
\hline acenaphthylene & $107 \pm 6$ & $106 \pm 5$ & $97 \pm 8$ & $103 \pm 3$ & $103 \pm 3$ & $108 \pm 3$ & $113 \pm 5$ & $115 \pm 4$ & $114 \pm 5$ \\
\hline acephate & $13 \pm 3$ & $\mathbf{1 1} \pm 1$ & $\mathbf{1 2} \pm 2$ & $\mathbf{1 8} \pm 4$ & $\mathbf{1 7} \pm 3$ & $\mathbf{1 4} \pm 2$ & $49 \pm 3$ & $\mathbf{2 9} \pm 2$ & $\mathbf{1 9} \pm 1$ \\
\hline alachlor & $93 \pm 17$ & $111 \pm 7$ & $108 \pm 7$ & $110 \pm 8$ & $105 \pm 3$ & $109 \pm 5$ & $118 \pm 8$ & $119 \pm 6$ & $118 \pm 7$ \\
\hline aldrin & $\mathbf{5 8} \pm 17$ & $80 \pm 13$ & $77 \pm 10$ & $80 \pm 10$ & $81 \pm 9$ & $83 \pm 8$ & $86 \pm 9$ & $78 \pm 15$ & $70 \pm 12$ \\
\hline ametryn & $100 \pm 8$ & $100 \pm 6$ & $93 \pm 7$ & $102 \pm 9$ & $98 \pm 3$ & $102 \pm 4$ & $110 \pm 7$ & $114 \pm 5$ & $108 \pm 6$ \\
\hline anthracene & $99 \pm 6$ & $103 \pm 4$ & $95 \pm 8$ & $94 \pm 9$ & $99 \pm 2$ & $103 \pm 5$ & $90 \pm 6$ & $107 \pm 1$ & $105 \pm 4$ \\
\hline atrazine & $101 \pm 9$ & $107 \pm 9$ & $96 \pm 8$ & $100 \pm 13$ & $102 \pm 7$ & $104 \pm 5$ & $106 \pm 10$ & $113 \pm 5$ & $109 \pm 7$ \\
\hline atrazine-desethyl & $91 \pm 12$ & $107 \pm 18$ & $99 \pm 9$ & $\mathbf{1 2 4} \pm 15$ & $108 \pm 7$ & $105 \pm 4$ & $108 \pm 18$ & $112 \pm 13$ & $109 \pm 8$ \\
\hline azinphos-ethyl & $118 \pm 9$ & $100 \pm 2$ & $92 \pm 8$ & $111 \pm 13$ & $104 \pm 4$ & $100 \pm 6$ & $127 \pm 3$ & $114 \pm 5$ & $103 \pm 7$ \\
\hline azinphos-methyl & $127 \pm 25$ & $106 \pm 15$ & $91 \pm 18$ & $108 \pm 19$ & $100 \pm 13$ & $87 \pm 9$ & $119 \pm 7$ & $87 \pm 6$ & $75 \pm 6$ \\
\hline azoxystrobin & $98 \pm 17$ & $78 \pm 6$ & $72 \pm 7$ & $0 \pm 0$ & $86 \pm 12$ & $80 \pm 13$ & $128 \pm 25$ & $103 \pm 16$ & $88 \pm 10$ \\
\hline benoxacor & $89 \pm 17$ & $110 \pm 7$ & $110 \pm 7$ & $110 \pm 5$ & $105 \pm 4$ & $110 \pm 4$ & $116 \pm 6$ & $120 \pm 7$ & $115 \pm 6$ \\
\hline benz(a)anthracene+chrysene ${ }^{a}$ & $102 \pm 8$ & $96 \pm 2$ & $84 \pm 7$ & $96 \pm 4$ & $99 \pm 4$ & $102 \pm 4$ & $99 \pm 4$ & $99 \pm 4$ & $98 \pm 4$ \\
\hline benzo(a)pyrene & $108 \pm 4$ & $112 \pm 2$ & $105 \pm 8$ & $101 \pm 8$ & $106 \pm 4$ & $113 \pm 4$ & $117 \pm 10$ & $124 \pm 8$ & $125 \pm 7$ \\
\hline benzo(b+k)fluoranthene & $96 \pm 6$ & $102 \pm 2$ & $94 \pm 7$ & $94 \pm 4$ & $99 \pm 5$ & $105 \pm 6$ & $102 \pm 4$ & $111 \pm 5$ & $112 \pm 7$ \\
\hline benzo(g,h,i)perylene & $104 \pm 7$ & $104 \pm 5$ & $94 \pm 5$ & $74 \pm 5$ & $94 \pm 3$ & $111 \pm 5$ & $100 \pm 11$ & $102 \pm 20$ & $104 \pm 10$ \\
\hline$\alpha-\mathrm{BHC}(\alpha-\mathrm{HCH})$ & $78 \pm 19$ & $101 \pm 9$ & $105 \pm 8$ & $103 \pm 6$ & $109 \pm 5$ & $109 \pm 6$ & $102 \pm 11$ & $105 \pm 13$ & $105 \pm 10$ \\
\hline$\beta-\mathrm{BHC}(\beta-\mathrm{HCH})$ & $95 \pm 8$ & $108 \pm 7$ & $103 \pm 8$ & $105 \pm 7$ & $103 \pm 4$ & $108 \pm 4$ & $103 \pm 8$ & $113 \pm 12$ & $111 \pm 10$ \\
\hline$\gamma$-BHC $(\gamma-\mathrm{HCH})$, lindane & $85 \pm 20$ & $111 \pm 7$ & $116 \pm 9$ & $101 \pm 10$ & $106 \pm 6$ & $107 \pm 5$ & $105 \pm 10$ & $107 \pm 11$ & $106 \pm 9$ \\
\hline$\delta-\mathrm{BHC}(\delta-\mathrm{HCH})$ & $89 \pm 8$ & $103 \pm 5$ & $101 \pm 7$ & $101 \pm 7$ & $101 \pm 4$ & $106 \pm 5$ & $101 \pm 10$ & $109 \pm 11$ & $107 \pm 10$ \\
\hline bifenthrin & $85 \pm 14$ & $84 \pm 6$ & $76 \pm 9$ & $88 \pm 7$ & $89 \pm 6$ & $93 \pm 5$ & $80 \pm 10$ & $81 \pm 13$ & $78 \pm 10$ \\
\hline
\end{tabular}




\begin{tabular}{|c|c|c|c|c|c|c|c|c|c|}
\hline bitertanol & $85 \pm 10$ & $78 \pm 4$ & $72 \pm 9$ & $79 \pm 14$ & $79 \pm 6$ & $72 \pm 7$ & $112 \pm 5$ & $102 \pm 6$ & $85 \pm 5$ \\
\hline bromophos & $82 \pm 10$ & $97 \pm 5$ & $94 \pm 8$ & $93 \pm 7$ & $93 \pm 4$ & $100 \pm 4$ & $104 \pm 9$ & $101 \pm 12$ & $96 \pm 10$ \\
\hline bromopropylate & $76 \pm 7$ & $76 \pm 4$ & $73 \pm 7$ & $81 \pm 10$ & $81 \pm 6$ & $79 \pm 6$ & $86 \pm 9$ & $86 \pm 13$ & $82 \pm 9$ \\
\hline bupirimate & $119 \pm 8$ & $106 \pm 3$ & $97 \pm 7$ & $100 \pm 7$ & $104 \pm 7$ & $106 \pm 3$ & $102 \pm 9$ & $99 \pm 6$ & $95 \pm 5$ \\
\hline buprofezin & $84 \pm 9$ & $99 \pm 8$ & $94 \pm 9$ & $101 \pm 11$ & $95 \pm 4$ & $102 \pm 6$ & $93 \pm 10$ & $102 \pm 14$ & $99 \pm 10$ \\
\hline cadusafos & $80 \pm 15$ & $114 \pm 8$ & $119 \pm 8$ & $99 \pm 4$ & $103 \pm 4$ & $107 \pm 4$ & $113 \pm 6$ & $116 \pm 6$ & $114 \pm 7$ \\
\hline carbofuran & $100 \pm 9$ & $109 \pm 5$ & $104 \pm 7$ & $105 \pm 5$ & $105 \pm 3$ & $111 \pm 4$ & $143 \pm 8$ & $134 \pm 5$ & $122 \pm 6$ \\
\hline carbophenothion & $91 \pm 17$ & $94 \pm 7$ & $90 \pm 10$ & $100 \pm 11$ & $95 \pm 5$ & $101 \pm 5$ & $105 \pm 10$ & $97 \pm 15$ & $89 \pm 10$ \\
\hline carboxin & $90 \pm 7$ & $89 \pm 5$ & $83 \pm 7$ & $89 \pm 7$ & $89 \pm 6$ & $90 \pm 6$ & $111 \pm 4$ & $114 \pm 6$ & $106 \pm 5$ \\
\hline carfentrazone-ethyl & $100 \pm 14$ & $107 \pm 8$ & $99 \pm 6$ & $97 \pm 9$ & $104 \pm 11$ & $107 \pm 5$ & $118 \pm 11$ & $119 \pm 5$ & $115 \pm 5$ \\
\hline cis-chlordane & $83 \pm 11$ & $88 \pm 7$ & $84 \pm 9$ & $92 \pm 10$ & $85 \pm 7$ & $89 \pm 7$ & $87 \pm 15$ & $87 \pm 17$ & $83 \pm 12$ \\
\hline trans-chlordane & $76 \pm 13$ & $89 \pm 5$ & $84 \pm 11$ & $93 \pm 13$ & $86 \pm 9$ & $87 \pm 7$ & $73 \pm 17$ & $88 \pm 16$ & $99 \pm 15$ \\
\hline chlorfenvinphos & $96 \pm 4$ & $95 \pm 3$ & $91 \pm 8$ & $97 \pm 4$ & $97 \pm 4$ & $97 \pm 4$ & $116 \pm 2$ & $115 \pm 5$ & $109 \pm 6$ \\
\hline chloroneb & $50 \pm 24$ & $118 \pm 20$ & $141 \pm 12$ & $77 \pm 11$ & $112 \pm 9$ & $116 \pm 11$ & $108 \pm 21$ & $115 \pm 14$ & $113 \pm 8$ \\
\hline chlorothalonil & $\mathbf{1} \pm 1$ & $3 \pm 3$ & $\mathbf{1 0} \pm 5$ & $18 \pm 3$ & $6 \pm 1$ & $2 \pm 0$ & $25 \pm 1$ & $\mathbf{1 0} \pm 0$ & $\mathbf{3} \pm 0$ \\
\hline chlorpropham & $103 \pm 14$ & $107 \pm 6$ & $101 \pm 8$ & $104 \pm 7$ & $103 \pm 4$ & $107 \pm 5$ & $114 \pm 6$ & $114 \pm 6$ & $110 \pm 8$ \\
\hline chlorpyrifos & $95 \pm 9$ & $109 \pm 4$ & $103 \pm 8$ & $107 \pm 7$ & $102 \pm 6$ & $106 \pm 4$ & $103 \pm 12$ & $105 \pm 14$ & $102 \pm 11$ \\
\hline chlorpyrifos-methyl & $83 \pm 10$ & $108 \pm 5$ & $109 \pm 10$ & $100 \pm 5$ & $100 \pm 5$ & $107 \pm 4$ & $108 \pm 8$ & $109 \pm 10$ & $106 \pm 9$ \\
\hline clofentezine & $126 \pm 11$ & $100 \pm 5$ & $89 \pm 8$ & $119 \pm 20$ & $100 \pm 18$ & $100 \pm 16$ & $133 \pm 9$ & $106 \pm 12$ & $101 \pm 9$ \\
\hline coumaphos & $117 \pm 12$ & $108 \pm 8$ & $95 \pm 8$ & $98 \pm 17$ & $107 \pm 6$ & $103 \pm 7$ & $113 \pm 4$ & $113 \pm 13$ & $107 \pm 8$ \\
\hline cyfluthrin & $97 \pm 28$ & $99 \pm 12$ & $95 \pm 12$ & $68 \pm 32$ & $100 \pm 13$ & $90 \pm 7$ & $65 \pm 41$ & $79 \pm \mathbf{2 4}$ & $93 \pm 12$ \\
\hline$\lambda$-cyhalothrin & $86 \pm 14$ & $95 \pm 13$ & $88 \pm 12$ & $96 \pm 13$ & $99 \pm 13$ & $91 \pm 5$ & $118 \pm 10$ & $114 \pm 14$ & $96 \pm 9$ \\
\hline cypermethrin & $107 \pm 13$ & $102 \pm 4$ & $90 \pm 8$ & $105 \pm 11$ & $100 \pm 5$ & $92 \pm 8$ & $105 \pm 8$ & $100 \pm 14$ & $93 \pm 10$ \\
\hline cyprodinil & $94 \pm 10$ & $98 \pm 8$ & $91 \pm 7$ & $85 \pm 12$ & $87 \pm 6$ & $97 \pm 5$ & $106 \pm 14$ & $102 \pm 11$ & $95 \pm 9$ \\
\hline$o, p^{\prime}-\mathrm{DDD}$ & $85 \pm 9$ & $93 \pm 6$ & $88 \pm 9$ & $93 \pm 6$ & $93 \pm 5$ & $96 \pm 5$ & $97 \pm 11$ & $94 \pm 13$ & $88 \pm 12$ \\
\hline$o, p^{\prime}-\mathrm{DDE}$ & $72 \pm 11$ & $81 \pm 8$ & $78 \pm 9$ & $99 \pm 13$ & $89 \pm 7$ & $89 \pm 6$ & $76 \pm 20$ & $99 \pm \mathbf{2 1}$ & $105 \pm 17$ \\
\hline$p, p^{\prime}$-DDE & $64 \pm 13$ & $65 \pm 24$ & $67 \pm 8$ & $83 \pm 6$ & $71 \pm 24$ & $82 \pm 7$ & $67 \pm 11$ & $67 \pm 11$ & $66 \pm 10$ \\
\hline$o, p^{\prime}-\mathrm{DDT}+p, p^{\prime}-\mathrm{DDD}^{\mathrm{a}}$ & $81 \pm 9$ & $84 \pm 6$ & $78 \pm 8$ & $88 \pm 7$ & $85 \pm 5$ & $91 \pm 6$ & $80 \pm 10$ & $80 \pm 14$ & $79 \pm 12$ \\
\hline$p, p^{\prime}$-DDT & $83 \pm 11$ & $79 \pm 7$ & $73 \pm 8$ & $84 \pm 7$ & $82 \pm 6$ & $88 \pm 6$ & $75 \pm 12$ & $73 \pm 14$ & $71 \pm 11$ \\
\hline
\end{tabular}




\begin{tabular}{|c|c|c|c|c|c|c|c|c|c|}
\hline dechlorane 604 & $<\mathrm{LCL}$ & $<\mathrm{LCL}$ & $82 \pm 23$ & $<\mathrm{LCL}$ & $<\mathrm{LCL}$ & $103 \pm 15$ & $<\mathrm{LCL}$ & $83 \pm 27$ & $89 \pm 14$ \\
\hline dechlorane plus, anti ${ }^{\mathbf{b}}$ & $97 \pm 13$ & $104 \pm 15$ & $97 \pm 14$ & $90 \pm \mathbf{4 5}$ & $104 \pm 11$ & $122 \pm 13$ & $117 \pm 17$ & $112 \pm 24$ & $113 \pm 12$ \\
\hline dechlorane plus, syn ${ }^{\mathbf{c}}$ & $66 \pm 16$ & $91 \pm 11$ & $93 \pm 14$ & $109 \pm 38$ & $104 \pm 13$ & $109 \pm 11$ & $84 \pm 16$ & $98 \pm 16$ & $107 \pm 10$ \\
\hline deltamethrin & $99 \pm 10$ & $92 \pm 7$ & $87 \pm 8$ & $107 \pm 27$ & $99 \pm 8$ & $95 \pm 11$ & $108 \pm 9$ & $98 \pm 14$ & $88 \pm 10$ \\
\hline dibenz(a,h)anthracene & $110 \pm 9$ & $110 \pm 9$ & $92 \pm 13$ & $89 \pm 12$ & $93 \pm 8$ & $104 \pm 11$ & $98 \pm 12$ & $82 \pm 43$ & $99 \pm 32$ \\
\hline dicloran & $95 \pm 8$ & $100 \pm 3$ & $99 \pm 6$ & $102 \pm 7$ & $95 \pm 5$ & $102 \pm 5$ & $130 \pm 5$ & $115 \pm 5$ & $106 \pm 6$ \\
\hline dicrotophos & $63 \pm 11$ & $46 \pm 4$ & $40 \pm 5$ & $82 \pm 30$ & $\mathbf{5 2} \pm 9$ & $43 \pm 4$ & $101 \pm 13$ & $70 \pm 5$ & $\mathbf{5 4} \pm 2$ \\
\hline dieldrin & $78 \pm 10$ & $92 \pm 8$ & $87 \pm 10$ & $97 \pm 12$ & $90 \pm 6$ & $93 \pm 5$ & $87 \pm 12$ & $89 \pm 11$ & $84 \pm 14$ \\
\hline difenoconazole & $103 \pm 10$ & $95 \pm 5$ & $86 \pm 7$ & $87 \pm 27$ & $90 \pm 5$ & $93 \pm 10$ & $119 \pm 6$ & $108 \pm 6$ & $102 \pm 6$ \\
\hline 2,6-diisopropylnaphthalene & $74 \pm 17$ & $96 \pm 14$ & $92 \pm 12$ & $75 \pm 6$ & $78 \pm 7$ & $83 \pm 7$ & $62 \pm 12$ & $67 \pm 15$ & $68 \pm 11$ \\
\hline dimethoate & $100 \pm 9$ & $107 \pm 5$ & $98 \pm 10$ & $97 \pm 7$ & $97 \pm 6$ & $91 \pm 29$ & $126 \pm 11$ & $109 \pm 7$ & $103 \pm 7$ \\
\hline diphenylamine & $87 \pm 13$ & $108 \pm 5$ & $109 \pm 9$ & $105 \pm 4$ & $101 \pm 4$ & $104 \pm 5$ & $110 \pm 6$ & $112 \pm 7$ & $108 \pm 9$ \\
\hline diuron & $133 \pm 16$ & $99 \pm 13$ & $70 \pm 14$ & $\mathbf{1 2 8} \pm 15$ & $86 \pm 9$ & $101 \pm 14$ & $122 \pm 25$ & $91 \pm 20$ & $68 \pm 6$ \\
\hline$\alpha$-endosulfan & $73 \pm 18$ & $94 \pm 13$ & $90 \pm 10$ & $98 \pm 5$ & $89 \pm 11$ & $94 \pm 5$ & $83 \pm 20$ & $87 \pm 16$ & $85 \pm 11$ \\
\hline$\beta$-endosulfan & $102 \pm 19$ & $107 \pm 9$ & $104 \pm 9$ & $108 \pm 6$ & $110 \pm 5$ & $109 \pm 7$ & $109 \pm 19$ & $116 \pm 9$ & $112 \pm 6$ \\
\hline endosulfan sulfate & $89 \pm 37$ & $104 \pm 17$ & $105 \pm 12$ & $106 \pm 44$ & $102 \pm 18$ & $109 \pm 16$ & $108 \pm 28$ & $\mathbf{1 2 3} \pm 18$ & $115 \pm 7$ \\
\hline endrin & $83 \pm 16$ & $95 \pm 9$ & $91 \pm 9$ & $95 \pm 7$ & $89 \pm 4$ & $94 \pm 7$ & $95 \pm 15$ & $94 \pm 15$ & $89 \pm 11$ \\
\hline endrin ketone & $107 \pm 8$ & $100 \pm 7$ & $99 \pm 11$ & $115 \pm 18$ & $100 \pm 12$ & $105 \pm 9$ & $100 \pm 12$ & $118 \pm 8$ & $106 \pm 10$ \\
\hline ethalfluralin & $67 \pm 24$ & $104 \pm 15$ & $115 \pm 11$ & $109 \pm 10$ & $116 \pm 7$ & $113 \pm 6$ & $111 \pm 18$ & $116 \pm 16$ & $107 \pm 9$ \\
\hline ethofumesate & $100 \pm 10$ & $111 \pm 4$ & $106 \pm 8$ & $107 \pm 7$ & $107 \pm 5$ & $111 \pm 5$ & $\mathbf{1 2 7} \pm 11$ & $135 \pm 8$ & $122 \pm 7$ \\
\hline ethoprop & $82 \pm 18$ & $105 \pm 18$ & $113 \pm 10$ & $93 \pm 14$ & $91 \pm 6$ & $96 \pm 7$ & $118 \pm 14$ & $107 \pm 7$ & $112 \pm 5$ \\
\hline ethoxyquin & $62 \pm 8$ & $74 \pm 8$ & $85 \pm 13$ & $88 \pm 19$ & $70 \pm 9$ & $91 \pm 10$ & $\mathbf{5 8} \pm 8$ & $75 \pm 15$ & $95 \pm 9$ \\
\hline fenarimol & $78 \pm 18$ & $74 \pm 5$ & $70 \pm 10$ & $75 \pm 11$ & $77 \pm 6$ & $69 \pm 6$ & $118 \pm 5$ & $102 \pm 8$ & $77 \pm 4$ \\
\hline fenazaquin & $85 \pm 12$ & $81 \pm 5$ & $73 \pm 7$ & $81 \pm 10$ & $80 \pm 7$ & $78 \pm 4$ & $91 \pm 10$ & $89 \pm 13$ & $73 \pm 10$ \\
\hline fenobucarb & $94 \pm 13$ & $114 \pm 7$ & $115 \pm 8$ & $107 \pm 6$ & $107 \pm 4$ & $110 \pm 3$ & $127 \pm 7$ & $125 \pm 4$ & $120 \pm 6$ \\
\hline fenpropathrin & $97 \pm 16$ & $96 \pm 5$ & $88 \pm 10$ & $111 \pm 14$ & $101 \pm 7$ & $94 \pm 4$ & $96 \pm 9$ & $100 \pm 11$ & $96 \pm 10$ \\
\hline fensulfothion & $110 \pm 6$ & $94 \pm 5$ & $85 \pm 8$ & $91 \pm 14$ & $92 \pm 4$ & $99 \pm 7$ & $120 \pm 6$ & $110 \pm 8$ & $99 \pm 6$ \\
\hline fenthion & $94 \pm 4$ & $105 \pm 4$ & $102 \pm 8$ & $103 \pm 6$ & $105 \pm 3$ & $111 \pm 5$ & $118 \pm 5$ & $118 \pm 6$ & $112 \pm 6$ \\
\hline fenvalerate + esfenvalerate ${ }^{\mathbf{a}}$ & $101 \pm 12$ & $94 \pm 5$ & $87 \pm 7$ & $103 \pm 21$ & $96 \pm 6$ & $95 \pm 10$ & $101 \pm 10$ & $97 \pm 13$ & $94 \pm 11$ \\
\hline
\end{tabular}




\begin{tabular}{|c|c|c|c|c|c|c|c|c|c|}
\hline fipronil & $104 \pm 9$ & $109 \pm 3$ & $104 \pm 9$ & $111 \pm 17$ & $105 \pm 8$ & $102 \pm 10$ & $134 \pm 7$ & $131 \pm 8$ & $118 \pm 7$ \\
\hline fipronil sulfide & $116 \pm 14$ & $117 \pm 8$ & $104 \pm 8$ & $112 \pm 14$ & $111 \pm 7$ & $112 \pm 3$ & $114 \pm 11$ & $117 \pm 6$ & $118 \pm 5$ \\
\hline fludioxonil & $113 \pm 9$ & $106 \pm 7$ & $96 \pm 7$ & $105 \pm 9$ & $106 \pm 4$ & $104 \pm 5$ & $123 \pm 8$ & $109 \pm 36$ & $113 \pm 6$ \\
\hline fluopyram & $104 \pm 11$ & $110 \pm 5$ & $100 \pm 8$ & $108 \pm 8$ & $104 \pm 7$ & $110 \pm 5$ & $128 \pm 9$ & $124 \pm 4$ & $115 \pm 5$ \\
\hline fluoranthene & $105 \pm 2$ & $104 \pm 3$ & $96 \pm 7$ & $100 \pm 3$ & $100 \pm 2$ & $105 \pm 2$ & $111 \pm 5$ & $113 \pm 2$ & $111 \pm 4$ \\
\hline fluorene & $112 \pm 11$ & $105 \pm 6$ & $96 \pm 11$ & $111 \pm 6$ & $90 \pm 3$ & $97 \pm 9$ & $\mathbf{1 3 1} \pm 18$ & $109 \pm 12$ & $96 \pm 5$ \\
\hline fluridone & $111 \pm 9$ & $104 \pm 6$ & $92 \pm 8$ & $89 \pm 25$ & $97 \pm 9$ & $101 \pm 12$ & $116 \pm 6$ & $115 \pm 9$ & $108 \pm 7$ \\
\hline flutriafol & $24 \pm 4$ & $22 \pm 1$ & $25 \pm 4$ & $26 \pm 10$ & $28 \pm 5$ & $26 \pm 4$ & $44 \pm 6$ & $37 \pm 4$ & $37 \pm 3$ \\
\hline$\tau$-fluvalinate & $100 \pm 11$ & $93 \pm 5$ & $87 \pm 8$ & $103 \pm 24$ & $96 \pm 6$ & $95 \pm 10$ & $104 \pm 9$ & $99 \pm 14$ & $94 \pm 10$ \\
\hline $\mathrm{HCDBCO}$ & $104 \pm 25$ & $92 \pm 12$ & $90 \pm 8$ & $76 \pm 35$ & $114 \pm 34$ & $114 \pm 12$ & $122 \pm 14$ & $119 \pm 11$ & $115 \pm 5$ \\
\hline heptachlor & $69 \pm 21$ & $87 \pm 12$ & $87 \pm 9$ & $85 \pm 9$ & $94 \pm 6$ & $92 \pm 8$ & $71 \pm 10$ & $79 \pm 16$ & $80 \pm 13$ \\
\hline heptachlor epoxide & $88 \pm 15$ & $100 \pm 9$ & $97 \pm 8$ & $107 \pm 16$ & $98 \pm 7$ & $100 \pm 5$ & $85 \pm 18$ & $95 \pm 13$ & $95 \pm 11$ \\
\hline heptenophos & $\mathbf{1 8} \pm 7$ & $35 \pm 5$ & $46 \pm 7$ & $49 \pm 5$ & $49 \pm 5$ & $45 \pm 6$ & $81 \pm 6$ & $72 \pm 3$ & $67 \pm 4$ \\
\hline hexabromobenzene (HBB) & $<\mathrm{LCL}$ & $<\mathrm{LCL}$ & $\mathbf{4 3} \pm 17$ & $<\mathrm{LCL}$ & $<\mathrm{LCL}$ & $63 \pm 14$ & $<\mathrm{LCL}$ & $33 \pm 30$ & $63 \pm 29$ \\
\hline hexachlorobenzene (HCB) & $78 \pm 22$ & $111 \pm 20$ & $112 \pm 19$ & $67 \pm 11$ & $86 \pm 9$ & $85 \pm 13$ & $46 \pm 10$ & $\mathbf{5 3} \pm 15$ & $56 \pm 11$ \\
\hline hexazinone & $102 \pm 6$ & $94 \pm 6$ & $88 \pm 7$ & $96 \pm 10$ & $97 \pm 5$ & $97 \pm 6$ & $101 \pm 9$ & $110 \pm 7$ & $112 \pm 6$ \\
\hline imazalil & $77 \pm 5$ & $73 \pm 5$ & $65 \pm 7$ & $31 \pm 9$ & $59 \pm 6$ & $61 \pm 8$ & $90 \pm 4$ & $77 \pm 4$ & $69 \pm 3$ \\
\hline indeno(1,2,3-c,d)pyrene & $91 \pm 9$ & $96 \pm 5$ & $85 \pm 7$ & $80 \pm 8$ & $89 \pm 5$ & $103 \pm 8$ & $90 \pm 6$ & $93 \pm 9$ & $96 \pm 4$ \\
\hline indoxacarb & $106 \pm 12$ & $103 \pm 8$ & $99 \pm 9$ & $92 \pm \mathbf{2 6}$ & $101 \pm 11$ & $102 \pm 11$ & $123 \pm 5$ & $\mathbf{1 3 0} \pm 13$ & $119 \pm 7$ \\
\hline iprodione & $106 \pm 11$ & $98 \pm 7$ & $88 \pm 9$ & $114 \pm 13$ & $100 \pm 10$ & $90 \pm 13$ & $\mathbf{1 4 4} \pm 11$ & $117 \pm 13$ & $100 \pm 5$ \\
\hline kresoxim-methyl & $108 \pm 8$ & $108 \pm 4$ & $101 \pm 8$ & $108 \pm 3$ & $108 \pm 4$ & $111 \pm 4$ & $125 \pm 6$ & $121 \pm 2$ & $116 \pm 5$ \\
\hline malathion & $101 \pm 6$ & $110 \pm 5$ & $107 \pm 8$ & $105 \pm 3$ & $105 \pm 4$ & $111 \pm 4$ & $125 \pm 3$ & $124 \pm 4$ & $115 \pm 4$ \\
\hline metalaxyl & $95 \pm 16$ & $105 \pm 5$ & $103 \pm 8$ & $101 \pm 11$ & $99 \pm 9$ & $108 \pm 6$ & $126 \pm 24$ & $127 \pm 8$ & $116 \pm 6$ \\
\hline methamidophos & $\mathbf{2 3} \pm 7$ & $\mathbf{1 0} \pm 1$ & $\mathbf{1} \pm 0$ & $13 \pm 2$ & $6 \pm 1$ & $\mathbf{3} \pm 1$ & $\mathbf{3 0} \pm 8$ & $12 \pm 3$ & $4 \pm 1$ \\
\hline methidathion & $106 \pm 5$ & $108 \pm 4$ & $101 \pm 8$ & $108 \pm 8$ & $106 \pm 3$ & $111 \pm 3$ & $118 \pm 2$ & $122 \pm 4$ & $116 \pm 6$ \\
\hline methiocarb & $89 \pm 7$ & $100 \pm 5$ & $99 \pm 7$ & $108 \pm 5$ & $102 \pm 3$ & $107 \pm 4$ & $106 \pm 4$ & $117 \pm 5$ & $113 \pm 6$ \\
\hline methoxychlor & $101 \pm 8$ & $96 \pm 4$ & $90 \pm 8$ & $100 \pm 6$ & $100 \pm 4$ & $102 \pm 6$ & $104 \pm 10$ & $102 \pm 11$ & $98 \pm 9$ \\
\hline metolachlor & $94 \pm 8$ & $109 \pm 4$ & $106 \pm 8$ & $105 \pm 5$ & $103 \pm 4$ & $108 \pm 4$ & $120 \pm 4$ & $123 \pm 5$ & $118 \pm 6$ \\
\hline metribuzin & $94 \pm 5$ & $101 \pm 3$ & $97 \pm 7$ & $103 \pm 6$ & $100 \pm 6$ & $105 \pm 4$ & $118 \pm 6$ & $118 \pm 5$ & $107 \pm 6$ \\
\hline
\end{tabular}




\begin{tabular}{|c|c|c|c|c|c|c|c|c|c|}
\hline mirex & $38 \pm 11$ & $54 \pm 8$ & $\mathbf{5 0} \pm 7$ & $66 \pm 8$ & $62 \pm 7$ & $64 \pm 7$ & $\mathbf{5 0} \pm 10$ & $53 \pm 12$ & $52 \pm 10$ \\
\hline myclobutanil & $98 \pm 8$ & $104 \pm 4$ & $100 \pm 7$ & $103 \pm 6$ & $105 \pm 4$ & $106 \pm 4$ & $117 \pm 4$ & $120 \pm 5$ & $114 \pm 5$ \\
\hline naphthalene & $114 \pm 8$ & $103 \pm 7$ & $100 \pm 16$ & $103 \pm 6$ & $105 \pm 8$ & $116 \pm 6$ & $102 \pm 17$ & $121 \pm 7$ & $120 \pm 7$ \\
\hline napropamide & $103 \pm 7$ & $106 \pm 5$ & $98 \pm 7$ & $105 \pm 3$ & $104 \pm 3$ & $107 \pm 4$ & $132 \pm 12$ & $115 \pm 7$ & $113 \pm 5$ \\
\hline cis-nonachlor & $72 \pm \mathbf{2 2}$ & $79 \pm 12$ & $82 \pm 11$ & $79 \pm 18$ & $83 \pm 10$ & $85 \pm 7$ & $58 \pm 30$ & $80 \pm 18$ & $79 \pm 14$ \\
\hline trans-nonachlor & $72 \pm 17$ & $86 \pm 11$ & $80 \pm 10$ & $80 \pm 20$ & $89 \pm 10$ & $90 \pm 6$ & $84 \pm 19$ & $82 \pm 14$ & $78 \pm 12$ \\
\hline omethoate & $22 \pm 10$ & $\mathbf{1 7} \pm 2$ & $\mathbf{1 9} \pm 4$ & $27 \pm 7$ & $\mathbf{1 7} \pm 4$ & $20 \pm 3$ & $32 \pm 13$ & $29 \pm 9$ & $26 \pm 3$ \\
\hline oxadixyl & $101 \pm 8$ & $101 \pm 6$ & $92 \pm 10$ & $103 \pm 11$ & $103 \pm 6$ & $99 \pm 5$ & $121 \pm 6$ & $121 \pm 5$ & $106 \pm 6$ \\
\hline oxyfluorfen & $112 \pm 14$ & $99 \pm 4$ & $92 \pm 6$ & $110 \pm 13$ & $108 \pm 6$ & $111 \pm 5$ & $112 \pm 8$ & $109 \pm 11$ & $105 \pm 8$ \\
\hline parathion-methyl & $99 \pm 17$ & $97 \pm 8$ & $100 \pm 12$ & $100 \pm 15$ & $102 \pm 9$ & $103 \pm 8$ & $105 \pm 15$ & $112 \pm 12$ & $109 \pm 9$ \\
\hline PBB 153 & $78 \pm 19$ & $90 \pm 10$ & $81 \pm 14$ & $86 \pm 14$ & $86 \pm 16$ & $85 \pm 10$ & $69 \pm 14$ & $93 \pm 18$ & $91 \pm 15$ \\
\hline PBDE 28 & $38 \pm 5$ & $\mathbf{5 7} \pm 12$ & $61 \pm 8$ & $53 \pm 11$ & $60 \pm 9$ & $73 \pm 8$ & $40 \pm 9$ & $\mathbf{5 0} \pm 15$ & $56 \pm 12$ \\
\hline PBDE 47 & $44 \pm 9$ & $\mathbf{5 1} \pm 12$ & $56 \pm 9$ & $77 \pm 10$ & $66 \pm 6$ & $69 \pm 7$ & $\mathbf{5 2} \pm 11$ & $56 \pm 13$ & $\mathbf{5 3} \pm 10$ \\
\hline PBDE 99 & $96 \pm 18$ & $107 \pm 13$ & $106 \pm 13$ & $106 \pm \mathbf{2 3}$ & $108 \pm 8$ & $112 \pm 12$ & $120 \pm 19$ & $117 \pm 19$ & $106 \pm 11$ \\
\hline PBDE 100 & $111 \pm 16$ & $116 \pm 18$ & $108 \pm 16$ & $122 \pm 20$ & $117 \pm 12$ & $117 \pm 15$ & $91 \pm \mathbf{2 3}$ & $118 \pm 22$ & $\mathbf{1 2 1} \pm 19$ \\
\hline PBDE 153 & $83 \pm 25$ & $95 \pm 18$ & $94 \pm 12$ & $85 \pm 24$ & $92 \pm 9$ & $95 \pm 15$ & $87 \pm 24$ & $95 \pm 18$ & $96 \pm 10$ \\
\hline PBDE 154 & $95 \pm 24$ & $95 \pm 13$ & $89 \pm 10$ & $98 \pm 19$ & $92 \pm 17$ & $93 \pm 12$ & $78 \pm \mathbf{3 2}$ & $95 \pm 17$ & $102 \pm 15$ \\
\hline PBDE 183 & $<\mathrm{LCL}$ & $<\mathrm{LCL}$ & $94 \pm 24$ & $<\mathrm{LCL}$ & $<\mathrm{LCL}$ & $88 \pm 14$ & $<\mathrm{LCL}$ & $86 \pm 18$ & $93 \pm 20$ \\
\hline PBEB & $43 \pm 17$ & $41 \pm 12$ & $39 \pm 7$ & $\mathbf{0} \pm 0$ & $36 \pm 9$ & $47 \pm 8$ & $28 \pm 13$ & $35 \pm 13$ & $36 \pm 9$ \\
\hline PBT & $38 \pm 16$ & $48 \pm 9$ & $44 \pm 5$ & $52 \pm 12$ & $47 \pm 6$ & $50 \pm 6$ & $33 \pm 13$ & $39 \pm 12$ & $40 \pm 11$ \\
\hline PCB 77 & $26 \pm 5$ & $\mathbf{5 1} \pm 10$ & $56 \pm 7$ & $49 \pm 13$ & $64 \pm 10$ & $76 \pm 9$ & $42 \pm 13$ & $\mathbf{5 2} \pm 14$ & $\mathbf{5 8} \pm 12$ \\
\hline PCB 81 & $23 \pm 7$ & $\mathbf{4 5} \pm 11$ & $54 \pm 8$ & $43 \pm 7$ & $\mathbf{5 5} \pm 9$ & $74 \pm 7$ & $32 \pm 10$ & $\mathbf{5 1} \pm 14$ & $56 \pm 12$ \\
\hline PCB 105 & $26 \pm 8$ & $\mathbf{4 3} \pm 16$ & $\mathbf{5 0} \pm 8$ & $47 \pm 13$ & $\mathbf{6 1 \pm 1 3}$ & $70 \pm 11$ & $54 \pm 14$ & $\mathbf{5 3} \pm 14$ & $\mathbf{5 5} \pm 13$ \\
\hline PCB 114 & $31 \pm 12$ & $47 \pm 11$ & $48 \pm 15$ & $33 \pm 8$ & $54 \pm 11$ & $73 \pm 9$ & $44 \pm 8$ & $47 \pm 15$ & $\mathbf{5 5} \pm 12$ \\
\hline PCB $123+118^{d}$ & $25 \pm 8$ & $44 \pm 12$ & $45 \pm 6$ & $23 \pm 8$ & $49 \pm 10$ & $65 \pm 7$ & $29 \pm 9$ & $43 \pm 11$ & $49 \pm 11$ \\
\hline PCB 126 & $\mathbf{2 6} \pm 6$ & $43 \pm 11$ & $48 \pm 8$ & $\mathbf{3 1} \pm 7$ & $53 \pm 10$ & $\mathbf{6 5} \pm 8$ & $31 \pm 9$ & $49 \pm 13$ & $\mathbf{5 1} \pm 12$ \\
\hline PCB $156+157^{d}$ & $106 \pm 31$ & $102 \pm 17$ & $80 \pm 10$ & $104 \pm 19$ & $98 \pm 20$ & $116 \pm 23$ & $109 \pm 20$ & $135 \pm 26$ & $119 \pm 17$ \\
\hline PCB 167 & $82 \pm 20$ & $99 \pm 29$ & $75 \pm 12$ & $96 \pm 18$ & $87 \pm 28$ & $99 \pm \mathbf{2 4}$ & $109 \pm \mathbf{2 6}$ & $114 \pm 28$ & $111 \pm 18$ \\
\hline PCB 170 & $100 \pm 18$ & $96 \pm 18$ & $75 \pm 6$ & $88 \pm 24$ & $94 \pm 14$ & $113 \pm 19$ & $92 \pm 36$ & $130 \pm 29$ & $130 \pm 39$ \\
\hline
\end{tabular}




\begin{tabular}{|c|c|c|c|c|c|c|c|c|c|}
\hline PCB 180 & $105 \pm 28$ & $100 \pm 26$ & $69 \pm 9$ & $98 \pm 23$ & $95 \pm 19$ & $101 \pm 15$ & $97 \pm 35$ & $113 \pm 28$ & $122 \pm 30$ \\
\hline PCB 189 & $97 \pm 20$ & $98 \pm 20$ & $73 \pm 9$ & $85 \pm 13$ & $87 \pm 21$ & $96 \pm 15$ & $89 \pm 16$ & $112 \pm \mathbf{3 0}$ & $106 \pm \mathbf{2 9}$ \\
\hline penconazole & $91 \pm 5$ & $97 \pm 4$ & $92 \pm 7$ & $100 \pm 3$ & $95 \pm 4$ & $100 \pm 4$ & $117 \pm 2$ & $114 \pm 4$ & $105 \pm 6$ \\
\hline pendimethalin & $88 \pm 10$ & $97 \pm 5$ & $96 \pm 9$ & $99 \pm 6$ & $94 \pm 5$ & $100 \pm 6$ & $112 \pm 11$ & $100 \pm 13$ & $93 \pm 10$ \\
\hline penthiopyrad & $113 \pm 10$ & $110 \pm 5$ & $99 \pm 7$ & $108 \pm 13$ & $107 \pm 5$ & $110 \pm 5$ & $119 \pm 5$ & $119 \pm 6$ & $116 \pm 5$ \\
\hline permethrin (cis+trans) & $90 \pm 14$ & $85 \pm 5$ & $76 \pm 7$ & $88 \pm 9$ & $90 \pm 6$ & $87 \pm 7$ & $82 \pm 10$ & $83 \pm 14$ & $79 \pm 12$ \\
\hline phenanthrene & $105 \pm 4$ & $108 \pm 4$ & $99 \pm 6$ & $105 \pm 3$ & $102 \pm 3$ & $103 \pm 8$ & $125 \pm 9$ & $119 \pm 3$ & $106 \pm 4$ \\
\hline$o$-phenylphenol & $83 \pm 17$ & $92 \pm 11$ & $92 \pm 7$ & $93 \pm 15$ & $88 \pm 5$ & $89 \pm 5$ & $105 \pm 9$ & $107 \pm 8$ & $98 \pm 5$ \\
\hline phorate & $74 \pm \mathbf{2 1}$ & $119 \pm 15$ & $137 \pm 11$ & $103 \pm 6$ & $110 \pm 6$ & $111 \pm 6$ & $106 \pm 14$ & $110 \pm 14$ & $110 \pm 7$ \\
\hline phosmet & $117 \pm 12$ & $96 \pm 5$ & $88 \pm 11$ & $104 \pm 8$ & $96 \pm 5$ & $93 \pm 6$ & $107 \pm 5$ & $90 \pm 7$ & $85 \pm 6$ \\
\hline phosmet oxon & $47 \pm 18$ & $70 \pm 10$ & $78 \pm 7$ & $81 \pm 11$ & $91 \pm 10$ & $95 \pm 11$ & $100 \pm 10$ & $140 \pm 16$ & $119 \pm 5$ \\
\hline piperonyl butoxide & $105 \pm 9$ & $100 \pm 4$ & $92 \pm 7$ & $101 \pm 11$ & $103 \pm 4$ & $106 \pm 4$ & $107 \pm 5$ & $109 \pm 8$ & $106 \pm 8$ \\
\hline pirimiphos-methyl & $93 \pm 7$ & $107 \pm 3$ & $106 \pm 7$ & $105 \pm 5$ & $101 \pm 4$ & $108 \pm 4$ & $109 \pm 6$ & $112 \pm 6$ & $111 \pm 8$ \\
\hline prochloraz & $95 \pm 16$ & $83 \pm 7$ & $79 \pm 8$ & $93 \pm 23$ & $91 \pm 7$ & $84 \pm 6$ & $124 \pm 7$ & $112 \pm 8$ & $96 \pm 5$ \\
\hline procymidone & $106 \pm 13$ & $104 \pm 7$ & $100 \pm 8$ & $104 \pm 15$ & $107 \pm 10$ & $112 \pm 5$ & $113 \pm 10$ & $120 \pm 6$ & $113 \pm 6$ \\
\hline profenophos & $37 \pm 5$ & $37 \pm 2$ & $36 \pm 6$ & $43 \pm 6$ & $43 \pm 7$ & $44 \pm 6$ & $70 \pm 6$ & $63 \pm 9$ & $58 \pm 5$ \\
\hline promecarb & $100 \pm 9$ & $112 \pm 5$ & $108 \pm 8$ & $106 \pm 6$ & $105 \pm 4$ & $112 \pm 4$ & $135 \pm 5$ & $133 \pm 5$ & $124 \pm 6$ \\
\hline prometryn & $95 \pm 9$ & $102 \pm 6$ & $96 \pm 7$ & $94 \pm 8$ & $98 \pm 5$ & $103 \pm 4$ & $114 \pm 8$ & $113 \pm 8$ & $106 \pm 7$ \\
\hline propachlor & $14 \pm 9$ & $33 \pm 12$ & $46 \pm 10$ & $102 \pm 8$ & $100 \pm 5$ & $99 \pm 6$ & $96 \pm 11$ & $91 \pm 6$ & $89 \pm 9$ \\
\hline propanil & $97 \pm 12$ & $97 \pm 4$ & $91 \pm 8$ & $98 \pm 4$ & $96 \pm 5$ & $102 \pm 4$ & $111 \pm 3$ & $108 \pm 6$ & $102 \pm 5$ \\
\hline propargite & $101 \pm 12$ & $113 \pm 4$ & $100 \pm 8$ & $92 \pm 6$ & $108 \pm 4$ & $111 \pm 4$ & $99 \pm 9$ & $111 \pm 11$ & $113 \pm 8$ \\
\hline propazine & $98 \pm 5$ & $102 \pm 4$ & $99 \pm 8$ & $102 \pm 7$ & $101 \pm 4$ & $107 \pm 4$ & $118 \pm 6$ & $113 \pm 7$ & $109 \pm 6$ \\
\hline propetamphos & $\mathbf{1 3 0} \pm 15$ & $120 \pm 6$ & $107 \pm 7$ & $127 \pm 27$ & $111 \pm 5$ & $114 \pm 5$ & $137 \pm 21$ & $124 \pm 8$ & $117 \pm 5$ \\
\hline propham & $81 \pm 19$ & $\mathbf{1 2 3} \pm 11$ & $131 \pm 10$ & $103 \pm 6$ & $111 \pm 5$ & $110 \pm 5$ & $122 \pm 8$ & $124 \pm 9$ & $117 \pm 5$ \\
\hline propiconazole & $91 \pm 12$ & $96 \pm 4$ & $90 \pm 8$ & $99 \pm 8$ & $94 \pm 5$ & $97 \pm 3$ & $109 \pm 6$ & $111 \pm 5$ & $108 \pm 6$ \\
\hline propyzamide & $101 \pm 5$ & $109 \pm 3$ & $104 \pm 7$ & $108 \pm 4$ & $106 \pm 4$ & $108 \pm 5$ & $118 \pm 4$ & $119 \pm 2$ & $111 \pm 6$ \\
\hline pyraclostrobin & $99 \pm 13$ & $103 \pm 6$ & $99 \pm 9$ & $94 \pm \mathbf{2 6}$ & $105 \pm 7$ & $110 \pm 13$ & $115 \pm 9$ & $112 \pm 11$ & $114 \pm 8$ \\
\hline pyrene & $104 \pm 3$ & $112 \pm 2$ & $103 \pm 8$ & $106 \pm 3$ & $107 \pm 4$ & $112 \pm 4$ & $118 \pm 6$ & $119 \pm 3$ & $115 \pm 3$ \\
\hline pyridaben & $100 \pm 9$ & $93 \pm 4$ & $85 \pm 7$ & $94 \pm 13$ & $94 \pm 5$ & $92 \pm 7$ & $102 \pm 27$ & $99 \pm 14$ & $89 \pm 11$ \\
\hline
\end{tabular}




\begin{tabular}{|c|c|c|c|c|c|c|c|c|c|}
\hline pyrimethanil & $91 \pm 8$ & $99 \pm 3$ & $93 \pm 7$ & $100 \pm 4$ & $98 \pm 5$ & $101 \pm 5$ & $107 \pm 4$ & $106 \pm 8$ & $101 \pm 7$ \\
\hline pyriproxyfen & $99 \pm 11$ & $93 \pm 4$ & $85 \pm 8$ & $89 \pm 9$ & $96 \pm 5$ & $101 \pm 6$ & $94 \pm 10$ & $93 \pm 12$ & $90 \pm 11$ \\
\hline quintozene (PCNB) & $53 \pm 24$ & $101 \pm 18$ & $116 \pm 12$ & $89 \pm 12$ & $95 \pm 8$ & $94 \pm 10$ & $83 \pm 15$ & $88 \pm 15$ & $83 \pm 14$ \\
\hline resmethrin & $106 \pm 12$ & $92 \pm 10$ & $82 \pm 9$ & $88 \pm 8$ & $95 \pm 8$ & $98 \pm 5$ & $95 \pm 12$ & $95 \pm 14$ & $87 \pm 10$ \\
\hline TBB & $101 \pm 19$ & $96 \pm 12$ & $90 \pm 12$ & $92 \pm 20$ & $97 \pm 13$ & $93 \pm 10$ & $81 \pm \mathbf{2 3}$ & $102 \pm \mathbf{2 1}$ & $99 \pm 11$ \\
\hline TBCO & $85 \pm 15$ & $92 \pm 9$ & $93 \pm 9$ & $92 \pm 10$ & $90 \pm 6$ & $91 \pm 6$ & $91 \pm 16$ & $78 \pm 16$ & $85 \pm 14$ \\
\hline TBECH & $\mathbf{6 1 \pm 1 3}$ & $91 \pm 10$ & $92 \pm 10$ & $90 \pm 11$ & $88 \pm 8$ & $89 \pm 6$ & $84 \pm 18$ & $98 \pm 15$ & $84 \pm 14$ \\
\hline TBNPA & $88 \pm 14$ & $104 \pm 7$ & $104 \pm 8$ & $89 \pm 9$ & $100 \pm 8$ & $101 \pm 4$ & $117 \pm 11$ & $113 \pm 5$ & $107 \pm 6$ \\
\hline TBX & $26 \pm 13$ & $43 \pm 9$ & $43 \pm 6$ & $53 \pm 11$ & $\mathbf{5 0} \pm 9$ & $\mathbf{5 1} \pm 7$ & $38 \pm 12$ & $\mathbf{4 0} \pm 11$ & $37 \pm 9$ \\
\hline TCEP & $103 \pm 7$ & $109 \pm 7$ & $102 \pm 8$ & $108 \pm 7$ & $106 \pm 8$ & $108 \pm 4$ & $132 \pm 9$ & $122 \pm 3$ & $117 \pm 4$ \\
\hline TCPP & $106 \pm 16$ & $114 \pm 9$ & $106 \pm 8$ & $69 \pm 13$ & $100 \pm 26$ & $112 \pm 6$ & $\mathbf{1 3 5} \pm 13$ & $131 \pm 5$ & $\mathbf{1 2 5} \pm 6$ \\
\hline TDCPP & $98 \pm 14$ & $109 \pm 8$ & $103 \pm 8$ & $100 \pm 20$ & $99 \pm 7$ & $106 \pm 5$ & $111 \pm 15$ & $112 \pm 8$ & $115 \pm 5$ \\
\hline tebuconazole & $48 \pm 11$ & $45 \pm 5$ & $47 \pm 8$ & $56 \pm 13$ & $51 \pm 6$ & $48 \pm 6$ & $64 \pm 18$ & $66 \pm 7$ & $65 \pm 5$ \\
\hline terbufos & $88 \pm 15$ & $105 \pm 8$ & $105 \pm 7$ & $101 \pm 8$ & $104 \pm 4$ & $109 \pm 5$ & $103 \pm 12$ & $107 \pm 11$ & $106 \pm 10$ \\
\hline terbutylazine & $96 \pm 5$ & $106 \pm 3$ & $99 \pm 6$ & $96 \pm 6$ & $101 \pm 5$ & $105 \pm 4$ & $118 \pm 8$ & $115 \pm 8$ & $110 \pm 7$ \\
\hline tetrachlorvinphos & $62 \pm 5$ & $64 \pm 2$ & $65 \pm 7$ & $72 \pm 7$ & $71 \pm 6$ & $69 \pm 4$ & $120 \pm 4$ & $111 \pm 5$ & $96 \pm 5$ \\
\hline tetraconazole & $97 \pm 11$ & $104 \pm 6$ & $101 \pm 9$ & $104 \pm 11$ & $108 \pm 10$ & $109 \pm 4$ & $108 \pm 6$ & $117 \pm 4$ & $121 \pm 7$ \\
\hline thiamethoxam & $108 \pm 18$ & $86 \pm 17$ & $83 \pm 13$ & $69 \pm 35$ & $74 \pm 16$ & $96 \pm 9$ & $122 \pm 8$ & $88 \pm 10$ & $77 \pm 5$ \\
\hline thiobencarb & $94 \pm 6$ & $101 \pm 4$ & $94 \pm 6$ & $100 \pm 4$ & $99 \pm 3$ & $105 \pm 4$ & $108 \pm 9$ & $109 \pm 9$ & $103 \pm 9$ \\
\hline tolclofos-methyl & $84 \pm 9$ & $108 \pm 5$ & $110 \pm 8$ & $103 \pm 5$ & $101 \pm 3$ & $105 \pm 3$ & $106 \pm 9$ & $113 \pm 10$ & $110 \pm 9$ \\
\hline triadimefon & $98 \pm 7$ & $107 \pm 5$ & $101 \pm 8$ & $109 \pm 5$ & $106 \pm 3$ & $108 \pm 4$ & $125 \pm 7$ & $121 \pm 5$ & $117 \pm 5$ \\
\hline tribufos & $75 \pm 10$ & $72 \pm 6$ & $69 \pm 7$ & $82 \pm 11$ & $79 \pm 7$ & $78 \pm 5$ & $88 \pm 9$ & $86 \pm 14$ & $80 \pm 9$ \\
\hline trifloxystrobin & $106 \pm 9$ & $107 \pm 4$ & $98 \pm 8$ & $114 \pm 7$ & $110 \pm 4$ & $112 \pm 5$ & $116 \pm 4$ & $119 \pm 4$ & $115 \pm 4$ \\
\hline triflumizole & $100 \pm 7$ & $98 \pm 4$ & $94 \pm 7$ & $109 \pm 8$ & $103 \pm 6$ & $103 \pm 5$ & $124 \pm 6$ & $132 \pm 7$ & $127 \pm 7$ \\
\hline trifluralin & $66 \pm 29$ & $\mathbf{1 2 1} \pm 14$ & $144 \pm 14$ & $112 \pm 12$ & $108 \pm 4$ & $108 \pm 7$ & $106 \pm 10$ & $105 \pm 16$ & $103 \pm 10$ \\
\hline triphenyl phosphate (TPP) & $94 \pm 7$ & $90 \pm 7$ & $87 \pm 6$ & $84 \pm 21$ & $92 \pm 7$ & $105 \pm 8$ & $92 \pm 7$ & $112 \pm 11$ & $112 \pm 7$ \\
\hline vinclozolin & $92 \pm 14$ & $110 \pm 7$ & $106 \pm 6$ & $100 \pm 14$ & $105 \pm 7$ & $112 \pm 4$ & $123 \pm 9$ & $122 \pm 6$ & $117 \pm 8$ \\
\hline
\end{tabular}


PCB spiking levels: $1,2.5,10 \mathrm{ng} / \mathrm{g} ;{ }^{\mathbf{a}}$ - spiking levels: $20,50,200 \mathrm{ng} / \mathrm{g} ;{ }^{\mathbf{b}}$ - spiking levels: $7.5,18.75,75 \mathrm{ng} / \mathrm{g}{ }^{\mathbf{c}}$ - spiking levels: 2.5 , $6.25,25 \mathrm{ng} / \mathrm{g} ;{ }^{\mathbf{d}}$ - spiking levels: 2, 5, $20 \mathrm{ng} / \mathrm{g}$. Bold values show recoveries and SD that are outside of the accepted quality control criteria of $70-120 \%$ for recoveries and $>20 \%$ for SD.

Table 2. Average \%recoveries \pm standard deviations (SD), $n=10$ each level and matrix, in method validation of the 98 pesticide analytes in cattle, pork, and chicken muscle tissues at three spiking levels using UHPLC-MS/MS for analysis.

\begin{tabular}{|c|c|c|c|c|c|c|c|c|c|}
\hline \multirow[b]{2}{*}{ Analyte } & \multicolumn{3}{|c|}{ CATTLE } & \multicolumn{3}{|c|}{ CHICKEN } & \multicolumn{3}{|c|}{ PORK } \\
\hline & $10 \mathrm{ng} / \mathrm{g}$ & $25 \mathrm{ng} / \mathrm{g}$ & $100 \mathrm{ng} / \mathrm{g}$ & $10 \mathrm{ng} / \mathrm{g}$ & $25 \mathrm{ng} / \mathrm{g}$ & $100 \mathrm{ng} / \mathrm{g}$ & $10 \mathrm{ng} / \mathrm{g}$ & $25 \mathrm{ng} / \mathrm{g}$ & $100 \mathrm{ng} / \mathrm{g}$ \\
\hline acephate & $46 \pm 4$ & $47 \pm 2$ & $46 \pm 5$ & $47 \pm 3$ & $46 \pm 4$ & $49 \pm 4$ & $51 \pm 6$ & $54 \pm 9$ & $\mathbf{5 0} \pm 5$ \\
\hline acetamiprid & $103 \pm 12$ & $99 \pm 6$ & $95 \pm 12$ & $112 \pm 8$ & $97 \pm 10$ & $105 \pm 9$ & $115 \pm 14$ & $122 \pm 20$ & $113 \pm 10$ \\
\hline aldicarb sulfone & $99 \pm 9$ & $102 \pm 5$ & $100 \pm 11$ & $101 \pm 6$ & $98 \pm 10$ & $103 \pm 11$ & $108 \pm 14$ & $\mathbf{1 2 1} \pm 19$ & $114 \pm 12$ \\
\hline aldicarb sulfoxide & $70 \pm 7$ & $68 \pm 3$ & $68 \pm 7$ & $71 \pm 5$ & $71 \pm 6$ & $75 \pm 6$ & $84 \pm 12$ & $87 \pm 14$ & $81 \pm 8$ \\
\hline aldicarb & $96 \pm 10$ & $101 \pm 5$ & $96 \pm 11$ & $99 \pm 10$ & $96 \pm 9$ & $106 \pm 11$ & $106 \pm 15$ & $120 \pm 11$ & $120 \pm 11$ \\
\hline ametryn & $98 \pm 7$ & $100 \pm 8$ & & $102 \pm 11$ & $97 \pm 5$ & & $113 \pm 15$ & $\mathbf{1 2 1} \pm 16$ & $116 \pm 12$ \\
\hline atrazine & $102 \pm 11$ & $106 \pm 9$ & $95 \pm 15$ & $108 \pm 17$ & $105 \pm 18$ & $111 \pm 8$ & $115 \pm 19$ & $118 \pm 18$ & $116 \pm 13$ \\
\hline atrazine-desethyl & $97 \pm 13$ & $106 \pm 9$ & $97 \pm 13$ & $94 \pm 16$ & $85 \pm 14$ & $92 \pm 12$ & $102 \pm 19$ & $112 \pm 19$ & $105 \pm 12$ \\
\hline azinphos-ethyl & $96 \pm 18$ & $102 \pm 13$ & $108 \pm 10$ & $115 \pm \mathbf{2 1}$ & $108 \pm \mathbf{2 2}$ & $111 \pm 18$ & $115 \pm 20$ & $117 \pm 13$ & $\mathbf{1 2 1} \pm 13$ \\
\hline azinphos-methyl & $96 \pm 9$ & $114 \pm 18$ & $114 \pm 17$ & $95 \pm 16$ & $109 \pm \mathbf{2 3}$ & $107 \pm 13$ & $78 \pm 10$ & $109 \pm 11$ & $\mathbf{1 2 1} \pm 16$ \\
\hline azoxystrobin & $106 \pm 15$ & $103 \pm 21$ & $102 \pm 13$ & $115 \pm 7$ & $109 \pm 7$ & $115 \pm 13$ & $113 \pm 16$ & $\mathbf{1 2 7} \pm 12$ & $\mathbf{1 2 8} \pm 13$ \\
\hline bifenthrin & $<\mathrm{LCL}$ & $<\mathrm{LCL}$ & $82 \pm 24$ & $<\mathrm{LCL}$ & $<\mathrm{LCL}$ & $113 \pm 36$ & $<\mathrm{LCL}$ & $<\mathrm{LCL}$ & $93 \pm 43$ \\
\hline buprofezin & $100 \pm 6$ & $100 \pm 5$ & $94 \pm 12$ & $97 \pm 6$ & $94 \pm 6$ & $102 \pm 9$ & $101 \pm 17$ & $106 \pm 17$ & $101 \pm 12$ \\
\hline cadusafos & $102 \pm 8$ & $103 \pm 6$ & $97 \pm 13$ & $107 \pm 9$ & $106 \pm 9$ & $108 \pm 9$ & $119 \pm 12$ & $121 \pm 9$ & $120 \pm 12$ \\
\hline carbaryl & $107 \pm 11$ & $109 \pm 6$ & $103 \pm 14$ & $109 \pm 12$ & $103 \pm 7$ & $111 \pm 10$ & $120 \pm 13$ & $\mathbf{1 2 6} \pm 16$ & $120 \pm 14$ \\
\hline carbendazim & $82 \pm 5$ & $81 \pm 5$ & $77 \pm 9$ & $86 \pm 8$ & $80 \pm 7$ & $85 \pm 10$ & $98 \pm 13$ & $102 \pm 18$ & $94 \pm 11$ \\
\hline carbofuran & $100 \pm 10$ & $103 \pm 7$ & $99 \pm 10$ & $106 \pm 8$ & $103 \pm 8$ & $109 \pm 9$ & $\mathbf{1 2 1} \pm 12$ & $\mathbf{1 2 3} \pm 10$ & $\mathbf{1 2 3} \pm 14$ \\
\hline carboxin & $95 \pm 8$ & $95 \pm 5$ & $88 \pm 12$ & $110 \pm 10$ & $107 \pm 13$ & $113 \pm 10$ & $119 \pm 13$ & $126 \pm 20$ & $119 \pm 10$ \\
\hline carfentrazone-ethyl & $112 \pm 25$ & $109 \pm 17$ & $101 \pm 24$ & $108 \pm \mathbf{2 6}$ & $108 \pm \mathbf{2 3}$ & $113 \pm 24$ & $105 \pm 23$ & $128 \pm 23$ & $119 \pm 18$ \\
\hline
\end{tabular}




\begin{tabular}{|c|c|c|c|c|c|c|c|c|c|}
\hline chlorfenvinphos & $110 \pm 9$ & $114 \pm 8$ & $103 \pm 12$ & $109 \pm 12$ & $104 \pm 5$ & $110 \pm 7$ & $119 \pm 13$ & $120 \pm 6$ & $119 \pm 11$ \\
\hline chlorpyrifos oxon & $63 \pm 18$ & $76 \pm 11$ & $96 \pm 10$ & $62 \pm 14$ & $64 \pm 10$ & $86 \pm 15$ & $80 \pm 22$ & $95 \pm 11$ & $107 \pm \mathbf{2 1}$ \\
\hline chlorsulfuron & $41 \pm 8$ & $36 \pm 5$ & $37 \pm 6$ & $38 \pm 10$ & $\mathbf{3 3} \pm 7$ & $34 \pm 7$ & $41 \pm 12$ & $43 \pm 7$ & $39 \pm 6$ \\
\hline coumaphos oxon & $113 \pm 13$ & $120 \pm 11$ & $114 \pm 17$ & $101 \pm 12$ & $108 \pm 13$ & $114 \pm 15$ & $88 \pm 21$ & $125 \pm 15$ & $136 \pm 12$ \\
\hline cyprodinil & $83 \pm 18$ & $92 \pm 8$ & $90 \pm 10$ & $105 \pm 15$ & $92 \pm 10$ & $100 \pm 10$ & $100 \pm 23$ & $102 \pm 18$ & $99 \pm 13$ \\
\hline cyromazine & $\mathbf{3 3} \pm 2$ & $14 \pm 5$ & $15 \pm 4$ & $67 \pm 5$ & $29 \pm 13$ & $24 \pm 7$ & $<\mathrm{LCL}$ & $16 \pm 4$ & $19 \pm 2$ \\
\hline dicrotophos & $97 \pm 8$ & $95 \pm 5$ & $90 \pm 16$ & $90 \pm 6$ & $93 \pm 9$ & $96 \pm 7$ & $105 \pm 11$ & $111 \pm 15$ & $105 \pm 11$ \\
\hline diflubenzuron & $<\mathrm{LCL}$ & $<\mathrm{LCL}$ & $77 \pm 35$ & $<\mathrm{LCL}$ & $<\mathrm{LCL}$ & $<\mathrm{LCL}$ & $<\mathrm{LCL}$ & $<\mathrm{LCL}$ & $136 \pm 37$ \\
\hline dimethoate & $98 \pm 12$ & $97 \pm 8$ & $95 \pm 10$ & $100 \pm 8$ & $101 \pm 7$ & $102 \pm 7$ & $116 \pm 16$ & $120 \pm 19$ & $114 \pm 12$ \\
\hline diuron & $108 \pm 16$ & $95 \pm 13$ & $102 \pm 16$ & $94 \pm 33$ & $94 \pm 15$ & $106 \pm 20$ & $117 \pm 24$ & $116 \pm 17$ & $118 \pm 18$ \\
\hline ethofumesate & $102 \pm 45$ & $109 \pm 39$ & $129 \pm 19$ & $<\mathrm{LCL}$ & $<\mathrm{LCL}$ & $121 \pm 26$ & $101 \pm \mathbf{3 0}$ & $128 \pm 27$ & $124 \pm 32$ \\
\hline ethoprop & $108 \pm 13$ & $109 \pm 5$ & $98 \pm 11$ & $112 \pm 15$ & $108 \pm 10$ & $112 \pm 10$ & $89 \pm 11$ & $119 \pm 8$ & $124 \pm 10$ \\
\hline fenazaquin & $94 \pm 13$ & $97 \pm 6$ & $90 \pm 13$ & $111 \pm 18$ & $99 \pm 14$ & $89 \pm 11$ & $69 \pm 33$ & $87 \pm 14$ & $93 \pm 15$ \\
\hline fenobucarb & $101 \pm 11$ & $102 \pm 10$ & $98 \pm 13$ & $105 \pm 8$ & $101 \pm 10$ & $110 \pm 11$ & $119 \pm 12$ & $128 \pm 23$ & $126 \pm 15$ \\
\hline fensulfothion & $99 \pm 13$ & $104 \pm 9$ & $95 \pm 11$ & $108 \pm \mathbf{2 1}$ & $100 \pm 12$ & $104 \pm 15$ & $112 \pm 16$ & $122 \pm 20$ & $129 \pm 14$ \\
\hline flumetsulam & $\mathbf{5 5} \pm 6$ & $51 \pm 7$ & $48 \pm 7$ & $57 \pm 10$ & $\mathbf{5 3} \pm 9$ & $\mathbf{5 7} \pm 8$ & $69 \pm 10$ & $70 \pm 9$ & $\mathbf{6 5} \pm 6$ \\
\hline fluopyram & $99 \pm 8$ & $101 \pm 11$ & $97 \pm 12$ & $108 \pm 15$ & $95 \pm 9$ & $100 \pm 10$ & $101 \pm 20$ & $120 \pm 11$ & $\mathbf{1 2 4} \pm 11$ \\
\hline $\begin{array}{l}\text { fluroxypyr- } \\
\text { methylheptyl ester }\end{array}$ & $128 \pm 26$ & $105 \pm \mathbf{3 0}$ & $106 \pm 25$ & $<\mathrm{LCL}$ & $<\mathrm{LCL}$ & $121 \pm 33$ & $116 \pm 31$ & $111 \pm 20$ & $115 \pm 22$ \\
\hline flutriafol & $92 \pm 13$ & $96 \pm 8$ & $96 \pm 16$ & $101 \pm 16$ & $97 \pm 11$ & $108 \pm 12$ & $\mathbf{1 2 4} \pm 15$ & $\mathbf{1 2 8} \pm 15$ & $115 \pm 11$ \\
\hline$\tau$-fluvalinate & $<\mathrm{LCL}$ & $<\mathrm{LCL}$ & $132 \pm 36$ & $<\mathrm{LCL}$ & $<\mathrm{LCL}$ & $126 \pm 35$ & $<\mathrm{LCL}$ & $<\mathrm{LCL}$ & $<\mathrm{LCL}$ \\
\hline fluxapyroxad & $115 \pm 20$ & $101 \pm 28$ & $107 \pm 26$ & $108 \pm \mathbf{4 2}$ & $99 \pm 24$ & $110 \pm 26$ & $117 \pm 35$ & $127 \pm 27$ & $118 \pm 21$ \\
\hline heptenophos & $99 \pm 14$ & $103 \pm 7$ & $100 \pm 14$ & $108 \pm 17$ & $104 \pm 10$ & $114 \pm 11$ & $119 \pm 16$ & $\mathbf{1 2 4} \pm 10$ & $120 \pm 13$ \\
\hline hexythiazox & $<\mathrm{LCL}$ & $99 \pm 19$ & $115 \pm 27$ & $<\mathrm{LCL}$ & $77 \pm \mathbf{2 6}$ & $73 \pm 13$ & $<\mathrm{LCL}$ & $86 \pm 24$ & $89 \pm 14$ \\
\hline 3-hydroxycarbofuran & $99 \pm 12$ & $106 \pm 6$ & $102 \pm 10$ & $105 \pm 9$ & $91 \pm 5$ & $105 \pm 11$ & $114 \pm 16$ & $124 \pm 21$ & $116 \pm 11$ \\
\hline imazalil & $84 \pm 10$ & $92 \pm 8$ & $89 \pm 10$ & $105 \pm 19$ & $93 \pm 11$ & $107 \pm 9$ & $119 \pm 16$ & $119 \pm 22$ & $112 \pm 14$ \\
\hline imazaquin & $\mathbf{1 1} \pm 5$ & $8 \pm 4$ & $9 \pm 1$ & $12 \pm 5$ & $5 \pm 3$ & $9 \pm 2$ & $<\mathrm{LCL}$ & $10 \pm 5$ & $\mathbf{1 0} \pm 2$ \\
\hline imazethapyr & $7 \pm 2$ & $8 \pm 1$ & $8 \pm 1$ & $8 \pm 4$ & $6 \pm 1$ & $7 \pm 2$ & $6 \pm 2$ & $9 \pm 3$ & $9 \pm 2$ \\
\hline imidacloprid & $73 \pm 15$ & $97 \pm 9$ & $87 \pm 9$ & $80 \pm 14$ & $79 \pm 16$ & $82 \pm 15$ & $119 \pm 13$ & $115 \pm 19$ & $121 \pm 14$ \\
\hline
\end{tabular}




\begin{tabular}{|c|c|c|c|c|c|c|c|c|c|}
\hline indoxacarb & $98 \pm 17$ & $104 \pm 20$ & $100 \pm 20$ & $86 \pm 41$ & $107 \pm 18$ & $106 \pm 31$ & $116 \pm 25$ & $123 \pm 34$ & $128 \pm 15$ \\
\hline linuron & $91 \pm 29$ & $103 \pm 16$ & $95 \pm 22$ & $71 \pm 35$ & $76 \pm 32$ & $88 \pm 23$ & $90 \pm 25$ & $114 \pm 19$ & $119 \pm 14$ \\
\hline malathion & $97 \pm 14$ & $103 \pm 14$ & $105 \pm 17$ & $107 \pm 12$ & $105 \pm 14$ & $109 \pm 14$ & $107 \pm 9$ & $125 \pm 9$ & $126 \pm 14$ \\
\hline metalaxyl & $100 \pm 10$ & $106 \pm 6$ & $100 \pm 11$ & $107 \pm 13$ & $107 \pm 11$ & $111 \pm 7$ & $110 \pm 13$ & $120 \pm 22$ & $119 \pm 14$ \\
\hline methamidophos & $37 \pm 4$ & $41 \pm 2$ & $39 \pm 4$ & $42 \pm 5$ & $40 \pm 3$ & $44 \pm 4$ & $46 \pm 7$ & $46 \pm 7$ & $44 \pm 4$ \\
\hline methiocarb & $117 \pm 15$ & $110 \pm 12$ & $106 \pm 19$ & $117 \pm 17$ & $107 \pm 12$ & $114 \pm 8$ & $115 \pm 10$ & $\mathbf{1 2 7} \pm 18$ & $\mathbf{1 2 4} \pm 15$ \\
\hline methomyl & $90 \pm 9$ & $96 \pm 6$ & $96 \pm 12$ & $99 \pm 7$ & $92 \pm 7$ & $101 \pm 9$ & $120 \pm 13$ & $118 \pm 20$ & $111 \pm 13$ \\
\hline methoprene & $87 \pm 9$ & $95 \pm 11$ & $87 \pm 11$ & $93 \pm 18$ & $103 \pm 18$ & $109 \pm 9$ & $87 \pm 20$ & $98 \pm 25$ & $93 \pm 19$ \\
\hline methoxyfenozide & $103 \pm 15$ & $102 \pm 17$ & $109 \pm 14$ & $106 \pm 9$ & $104 \pm 15$ & $105 \pm 11$ & $123 \pm 9$ & $126 \pm 9$ & $126 \pm 14$ \\
\hline metolachlor & $99 \pm 8$ & $100 \pm 7$ & $92 \pm 14$ & $102 \pm 10$ & $102 \pm 5$ & $103 \pm 13$ & $106 \pm 11$ & $118 \pm 9$ & $121 \pm 7$ \\
\hline monocrotophos & $79 \pm 6$ & $80 \pm 6$ & $80 \pm 8$ & $87 \pm 8$ & $85 \pm 8$ & $90 \pm 7$ & $104 \pm 10$ & $108 \pm 17$ & $101 \pm 11$ \\
\hline norflurazon & $93 \pm 25$ & $95 \pm 14$ & $98 \pm 10$ & $116 \pm 20$ & $92 \pm 18$ & $111 \pm 13$ & $124 \pm 20$ & $124 \pm 22$ & $\mathbf{1 2 3} \pm 18$ \\
\hline novaluron & $<\mathrm{LCL}$ & $<\mathrm{LCL}$ & $120 \pm 22$ & $<\mathrm{LCL}$ & $<\mathrm{LCL}$ & $<\mathrm{LCL}$ & $<\mathrm{LCL}$ & $<\mathrm{LCL}$ & $123 \pm 26$ \\
\hline omethoate & $62 \pm 3$ & $64 \pm 3$ & $60 \pm 7$ & $65 \pm 7$ & $61 \pm 5$ & $64 \pm 5$ & $79 \pm 9$ & $80 \pm 13$ & \\
\hline oxadixyl & $103 \pm 8$ & $108 \pm 6$ & $100 \pm 12$ & $104 \pm 11$ & $103 \pm 9$ & $107 \pm 7$ & $115 \pm 10$ & $125 \pm 21$ & $118 \pm 11$ \\
\hline oxamyl & $98 \pm 8$ & $99 \pm 5$ & $97 \pm 11$ & $98 \pm 12$ & $94 \pm 11$ & $99 \pm 9$ & $112 \pm 15$ & $117 \pm 14$ & $110 \pm 9$ \\
\hline oxydemeton-methyl & $71 \pm 8$ & $77 \pm 7$ & $70 \pm 10$ & $78 \pm 12$ & $72 \pm 9$ & $79 \pm 9$ & $91 \pm 14$ & $100 \pm 12$ & $91 \pm 8$ \\
\hline paraoxon-ethyl & $107 \pm 18$ & $92 \pm 24$ & $92 \pm 24$ & $85 \pm 37$ & $82 \pm 16$ & $97 \pm 18$ & $107 \pm 25$ & $122 \pm 21$ & $\mathbf{1 2 4} \pm 19$ \\
\hline paraoxon-methyl & $91 \pm 28$ & $106 \pm 14$ & $95 \pm 11$ & $101 \pm 6$ & $71 \pm 13$ & $101 \pm 16$ & $110 \pm 23$ & $96 \pm 17$ & $111 \pm 18$ \\
\hline penconazole & $99 \pm 8$ & $97 \pm 10$ & $93 \pm 12$ & $119 \pm 25$ & $111 \pm 13$ & $\mathbf{1 2 1} \pm 16$ & $111 \pm 5$ & $128 \pm 19$ & $117 \pm 10$ \\
\hline penthiopyrad & $113 \pm 13$ & $111 \pm 8$ & $103 \pm 16$ & $131 \pm 14$ & $120 \pm 14$ & $119 \pm 12$ & $120 \pm 14$ & $120 \pm 15$ & $119 \pm 16$ \\
\hline permethrin & $<\mathrm{LCL}$ & $<\mathrm{LCL}$ & $<\mathrm{LCL}$ & $<\mathrm{LCL}$ & $<\mathrm{LCL}$ & $<\mathrm{LCL}$ & $<\mathrm{LCL}$ & $<\mathrm{LCL}$ & $<\mathrm{LCL}$ \\
\hline phosmet & $101 \pm 27$ & $89 \pm 17$ & $100 \pm 35$ & $113 \pm 33$ & $109 \pm 28$ & $150 \pm 26$ & $94 \pm 31$ & $119 \pm 25$ & $140 \pm 33$ \\
\hline phosmet oxon & $99 \pm 9$ & $100 \pm 4$ & $96 \pm 10$ & $107 \pm 13$ & $99 \pm 9$ & $111 \pm 7$ & $\mathbf{1 2 1} \pm 11$ & $120 \pm 9$ & $116 \pm 15$ \\
\hline pirimiphos-methyl & $102 \pm 9$ & $103 \pm 4$ & $98 \pm 13$ & $101 \pm 8$ & $96 \pm 8$ & $101 \pm 8$ & $110 \pm 17$ & $115 \pm 18$ & $111 \pm 11$ \\
\hline profenofos & $97 \pm 12$ & $94 \pm 13$ & $93 \pm 16$ & $99 \pm 17$ & $80 \pm 13$ & $90 \pm 11$ & $96 \pm 22$ & $107 \pm 16$ & $108 \pm 17$ \\
\hline promecarb & $110 \pm 8$ & $106 \pm 16$ & $103 \pm 13$ & $102 \pm 11$ & $104 \pm 11$ & $107 \pm 9$ & $119 \pm 15$ & $\mathbf{1 2 5} \pm 17$ & $121 \pm 11$ \\
\hline promethryn & $102 \pm 10$ & $92 \pm 20$ & $95 \pm 14$ & $106 \pm 9$ & $97 \pm 7$ & $105 \pm 9$ & $110 \pm 12$ & $119 \pm 16$ & $114 \pm 11$ \\
\hline propanil & $<\mathrm{LCL}$ & $75 \pm 12$ & $78 \pm 16$ & $<\mathrm{LCL}$ & $99 \pm 19$ & $111 \pm 17$ & $<\mathrm{LCL}$ & $129 \pm 28$ & $\mathbf{1 3 4} \pm 20$ \\
\hline
\end{tabular}




\begin{tabular}{|c|c|c|c|c|c|c|c|c|c|}
\hline propazine & $91 \pm 11$ & $104 \pm 10$ & $97 \pm 13$ & $107 \pm 13$ & $98 \pm 11$ & $111 \pm 11$ & $121 \pm 19$ & $128 \pm 32$ & $117 \pm 13$ \\
\hline propiconazole & $102 \pm 9$ & $99 \pm 7$ & $97 \pm 9$ & $106 \pm 16$ & $96 \pm 10$ & $102 \pm 13$ & $106 \pm 14$ & $116 \pm 10$ & $120 \pm 13$ \\
\hline propoxur & $102 \pm 8$ & $106 \pm 4$ & $102 \pm 12$ & $104 \pm 10$ & $101 \pm 9$ & $109 \pm 9$ & $115 \pm 12$ & $120 \pm 17$ & $117 \pm 11$ \\
\hline pymetrozine & $47 \pm 4$ & $48 \pm 4$ & $46 \pm 6$ & $47 \pm 5$ & $44 \pm 4$ & $49 \pm 5$ & $\mathbf{5 3} \pm 7$ & $\mathbf{5 3} \pm 8$ & $51 \pm 6$ \\
\hline pyraclostrobin & $103 \pm 8$ & $103 \pm 8$ & $102 \pm 13$ & $107 \pm 10$ & $99 \pm 7$ & $106 \pm 10$ & $120 \pm 11$ & $\mathbf{1 2 5} \pm 16$ & $119 \pm 11$ \\
\hline pyridaben & $88 \pm 7$ & $85 \pm 7$ & $82 \pm 12$ & $102 \pm 10$ & $94 \pm 8$ & $104 \pm 8$ & $92 \pm 15$ & $95 \pm 17$ & $94 \pm 12$ \\
\hline pyrimethanil & $98 \pm 10$ & $102 \pm 5$ & $95 \pm 9$ & $104 \pm 14$ & $94 \pm 8$ & $102 \pm 10$ & $\mathbf{1 2 1} \pm 14$ & $116 \pm 14$ & $111 \pm 11$ \\
\hline quizalofop-ethyl & $98 \pm 16$ & $100 \pm 17$ & $93 \pm 18$ & $111 \pm 29$ & $108 \pm \mathbf{3 2}$ & $115 \pm 23$ & $101 \pm 15$ & $110 \pm 18$ & $112 \pm 11$ \\
\hline resmethrin & $104 \pm 26$ & $119 \pm 37$ & $93 \pm 31$ & $<\mathrm{LCL}$ & $<\mathrm{LCL}$ & $101 \pm \mathbf{2 1}$ & $93 \pm 20$ & $88 \pm 34$ & $92 \pm 14$ \\
\hline spinosyn $\mathrm{A}^{\mathbf{a}}$ & $105 \pm 11$ & $106 \pm 8$ & $104 \pm 12$ & $110 \pm 8$ & $102 \pm 10$ & $111 \pm 10$ & $120 \pm 7$ & $\mathbf{1 2 3} \pm 10$ & $122 \pm 12$ \\
\hline spinosyn $\mathrm{D}^{\mathbf{b}}$ & $99 \pm 7$ & $102 \pm 7$ & $101 \pm 12$ & $108 \pm 9$ & $98 \pm 5$ & $108 \pm 8$ & $114 \pm 13$ & $125 \pm 21$ & $117 \pm 12$ \\
\hline tebuconazole & $107 \pm 15$ & $111 \pm 12$ & $105 \pm 13$ & $109 \pm 27$ & $93 \pm 15$ & $106 \pm 10$ & $124 \pm 17$ & $125 \pm 27$ & $121 \pm 14$ \\
\hline tebufenozide & $102 \pm 12$ & $102 \pm 7$ & $101 \pm 14$ & $113 \pm 11$ & $108 \pm 12$ & $109 \pm 10$ & $114 \pm 17$ & $120 \pm 11$ & $\mathbf{1 2 5} \pm 15$ \\
\hline temephos & $111 \pm 24$ & $100 \pm 14$ & $98 \pm 14$ & $97 \pm 34$ & $94 \pm 27$ & $113 \pm 17$ & $99 \pm 11$ & $117 \pm 9$ & $119 \pm 11$ \\
\hline tetrachlorvinphos & $117 \pm 23$ & $115 \pm 12$ & $105 \pm 11$ & $128 \pm 18$ & $98 \pm 24$ & $105 \pm 16$ & $113 \pm 11$ & $127 \pm 25$ & $123 \pm 15$ \\
\hline tetraconazole & $99 \pm 15$ & $109 \pm 13$ & $106 \pm 14$ & $105 \pm 37$ & $135 \pm 36$ & $124 \pm 32$ & $122 \pm 26$ & $\mathbf{1 2 8} \pm 14$ & $120 \pm 20$ \\
\hline thiabendazole & $89 \pm 7$ & $87 \pm 4$ & $82 \pm 9$ & $91 \pm 7$ & $87 \pm 10$ & $94 \pm 8$ & $104 \pm 11$ & $103 \pm 15$ & $97 \pm 11$ \\
\hline thiamethoxam & $89 \pm 7$ & $93 \pm 7$ & $84 \pm 8$ & $95 \pm 9$ & $97 \pm 11$ & $98 \pm 9$ & $109 \pm 10$ & $118 \pm 22$ & $107 \pm 13$ \\
\hline tolylfluanid & $11 \pm 0$ & $5 \pm 3$ & $8 \pm 4$ & $<\mathrm{LCL}$ & $<\mathrm{LCL}$ & $<\mathrm{LCL}$ & $<\mathrm{LCL}$ & $<\mathrm{LCL}$ & $<\mathrm{LCL}$ \\
\hline triadimefon & $105 \pm 12$ & $98 \pm 24$ & $105 \pm 16$ & $94 \pm 49$ & $107 \pm \mathbf{3 7}$ & $119 \pm 24$ & $121 \pm 26$ & $119 \pm 17$ & $123 \pm 16$ \\
\hline trifloxystrobin & $99 \pm 12$ & $97 \pm 11$ & $97 \pm 14$ & $114 \pm 13$ & $102 \pm 15$ & $108 \pm 11$ & $120 \pm 7$ & $122 \pm 7$ & $120 \pm 9$ \\
\hline trinexapac-ethyl & $108 \pm 29$ & $104 \pm 25$ & $84 \pm 15$ & $<\mathrm{LCL}$ & $78 \pm 34$ & $90 \pm 33$ & $113 \pm 31$ & $114 \pm 35$ & $112 \pm 24$ \\
\hline
\end{tabular}

${ }^{\mathrm{a}}$ - spiking levels: $8.5,21.25,85 \mathrm{ng} / \mathrm{g} ;{ }^{\mathbf{b}}-$ spiking levels: $1.5,3.75,15 \mathrm{ng} / \mathrm{g}$. Bold values show recoveries and SD that are outside of the accepted quality control criteria of $70-120 \%$ for recoveries and $>20 \%$ for SD. 
Table 3. Analytes determined in samples from the market.

\begin{tabular}{|c|c|c|c|}
\hline $\begin{array}{l}\text { Muscle } \\
\text { Sample }\end{array}$ & Analyte & $\begin{array}{c}\text { Concentration } \\
\text { (ng/g) }\end{array}$ & $\begin{array}{c}\text { Tolerance } \\
\text { (ng/g) }\end{array}$ \\
\hline \multirow{3}{*}{ Lamb } & $p, p^{\prime}-\mathrm{DDE}$ & $0.8 *$ & \\
\hline & $\alpha-\mathrm{BHC}$ & 1.5 & \\
\hline & tetraconazole & 5.7 & 10 \\
\hline \multirow{5}{*}{ Catfish } & $p, p^{\prime}-\mathrm{DDE}$ & 24 & \\
\hline & $o, p^{\prime}-\mathrm{DDD}$ & 1.1 & \\
\hline & $o, p^{\prime}-\mathrm{DDT}+p, p^{\prime}-\mathrm{DDD}$ & 9.3 & \\
\hline & $p, p^{\prime}$-DDT & $0.9 *$ & \\
\hline & metolachlor & $0.7 *$ & 20 \\
\hline Pork & piperonyl butoxide & 49 & 100 \\
\hline
\end{tabular}

$*<\mathrm{LCL}$ of $1 \mathrm{ng} / \mathrm{g}$ 